Niniejsza publikacja jest dostępna na licencji Creative Commons. Uznanie autorstwa-Użycie niekomercyjne-Bez utworów zależnych 3.0 Polska. Pewne prawa zastrzeżone na rzecz autora. Zezwala się na wykorzystanie publikacji zgodnie z licencja - pod warunkiem zachowania niniejszej informacji licencyjnej oraz wskazania autora jako właściciela praw do tekstu. Treść licencji jest dostępna na stronie: http://creativecommons.org/licenses/by-nc-nd/3.0/pl/

Lingwistyka Stosowana 24: 4/ 2017, 109-138

\author{
Beata MIKOLAJCZYK \\ Jarosław APTACY \\ Adam-Mickiewicz-Universität
}

\title{
Intratextuelle Verweise - Kohäsionsstiftung in Rechtstexten, dargestellt am Beispiel der Textsorte Habilitationsordnung
}

\begin{abstract}
:
Intratextual references - cohesion in legal texts, illustrated on the example of the text type habilitation regulation

In this article the form and function of the anaphoric and cataphoric intratextual references in a legal text are examined. Such references include, but are not limited to, the cohesion of a text and contribute to the understanding of the text. 29 habilitation regulations of various German universities are used as a basis for examination. References appear as phrases and dependent clauses, with prepositional phrases being most common. Heads of such phrases are usually formed by the prepositions gemäß (according to) and the synonymous nach, followed by phrases which are subject to the grammaticalization process, e.g. im Sinne (as defined by/ in the meaning of). Syntactically, such references function as attributes of different levels. Occasionally, adverbials occur as well. We consider the analyzed references to be a hallmark of legal texts, which are among the most important means of coherence. In the case of habilitation regulations, it must also be taken into account that readers usually choose certain text passages (text contents) according to their interests. In such a case, the references help to ensure that the necessary knowledge is conveyed relatively completely by means of references to other text parts, thereby achieving the individual reading goal of the recipient.
\end{abstract}

\section{Einleitung}

Jeder, der einen Rechtstext jeglicher Art in der Hand hatte, weiß, dass Verweise in Rechtstexten sehr häufig vorkommen und dass sie unabdingbar sind. In den Rechtswissenschaften wird in solchen Zusammenhängen oft von Verweisung, Bezugnahme und/ oder Inkorporation gesprochen. Es besteht kein Konsens darüber, wie die Beziehungen zwischen ihnen zu explizieren sind (hierzu vgl. M. Niedobitek 2001: 163). Manchmal wird die Inkorporation als eine Unterkategorie der Verweisung angesehen (vgl. L. Hertel/ B. Oberbichler/ Th. Wilrich 2015: 46). Gelegentlich werden sie (Inkorporation und Verweise) auseinandergehalten. Während ein Verweis eine Bezugnahme entweder auf einen anderen rechtssysteminternen Text oder auf eine Norm innerhalb eines Rechtstextes darstellt, versteht man unter Inkorporation die textliche Übernahme einer anderen Norm in den betreffenden Text. Juristisch gesehen fungieren die beiden Arten als Textverbindungen innerhalb eines Rechtstextnetzwerks. 
Im Lichte der sprachwissenschaftlichen Betrachtung hat man es dabei auch mit zwei Verfahren zu tun. Die Grenze verläuft nicht wie in der Rechtswissenschaft zwischen Verweisung schlechthin und Inkorporation, sondern sie wird durch die Abgrenzung eines Textes bestimmt und führt dazu, dass dabei mal intertextuelle Beziehungen durch sog. Binnenverweisungen hergestellt werden und mal intertextuelle durch Inkorporation und Außenverweisungen zustande kommen. Bei der Anwendung des modernen textlinguistischen Instrumentariums kann hier also zwischen der Textkohärenzstiftung und der Herstellung der Intertextualität differenziert werden. Die Herstellung der intertextuellen Bezüge wird wiederum durch zwei Verfahren erreicht, durch direkte Wiederaufnahme (Inkorporation) und Referenz (Außenverweisung).

Was in der Rechtswissenschaft oft für einen Aspekt der Rechtstexte (Verweisung) gehalten wird, wird in der (Text)Linguistik differenzierter gesehen. Der Beitrag stellt sich die Aufgabe, innertextuelle Verweisungen in Rechtstexten am Beispiel von Habilitationsordnungen auf der Folie der Kohäsions- und Kohärenzstiftung zu beschreiben und zu diskutieren.

Als ein Spezifikum von Rechtstexten gilt, dass ihre Kohärenz u.a. durch strukturelle Ordnung gewährleistet wird. Sie charakterisieren sich durch eine feine Gliederung, die den Textzusammenhalt stabil macht (vgl. R. de Beaugrande/ W.U. Dressler 1981) und die Lektüre des Textes optimalisieren soll. In diesem Beitrag wird ein struktureller Aspekt der Habilitationsordnung herausgegriffen und zum Gegenstand der Betrachtung gemacht, und zwar geht es um intratextuelle Bezüge. Schon auf den ersten Blick sieht man, dass sie eine schnelle und einfache Orientierung und "Bewegung" des Rezipienten im Text erleichtern. Nun wird gezeigt, wie sie linguistisch erfasst werden können. Wir befassen uns mit zwei Aspekten der Verweise, zum einem mit ihrer Form, zum anderen mit ihrer Leistung im Text.

\section{Die Bestimmung der Textsorte}

Ausgegangen wird von dem Textsortenkonzept nach K. Adamzik (1995: 24ff.), nach der Textsorten als Ausdruck von Textmustern, als kommunikative Routine gelten, mit der eine kommunikative Aufgabe gelöst wird. Eine Textsorte stellt ein etabliertes kommunikatives Muster dar, das in einem gegebenen Zeitraum einer Konvention, aber auch im Laufe der Zeit Wandlungen unterliegt. Bei einer solchen Betrachtung handelt es sich bei einer Habilitationsordnung nicht um eine eigenständige und abgrenzbare Textsorte, sondern sie ordnet sich neben verschiedene andere Ordnungen ein und kann demzufolge als eine Unterklasse der Textsortenkategorie Ordnung aufgefasst werden. Sie weist für diese Klasse typische Charakteristika auf, primär die Textfunktion (ihr normativ-bindender Charakter im juristischen Sinne) und sekundär den Textaufbau (für juristische Texte typische Gliederung). Sie unterscheidet sich von anderen Ordnungen jedoch durch den Handlungsraum, die behandelte Thematik und die für den Handlungsraum spezifische Lexik.

Die Kriterien für die Charakteristik einer Textsorte sind sehr heterogen und lassen sich auf verschiedenen Ebenen ansiedeln. Wir ziehen zu diesem Zweck drei Kriterien heran, situative Variablen, die Textfunktion und strukturelle (textuelle und sprachliche) Aspekte. Eine kurze Präsentation der Textsorte Habilitationsordnung $(=\mathrm{HO})$ kann man 
gerade mit einer Beschreibung von externen Merkmalen beginnen. Als Textproduzent gilt eine Institution, in diesem konkreten Fall eine Fakultät, die rechtlich gesehen dazu befugt ist, den Grad Doktor habil. in einer genau festgelegten Wissenschaftsdisziplin zu verleihen. Die Anzahl der Kommunikationsbeteiligten lässt sich kaum bestimmen, man kann jedoch prototypisch eine Gruppe von potenziellen Lesern annehmen, dazu gehören zweifelsohne die in das Habilitationsverfahren direkt Involvierten, d.h. Habilitationskandidaten, Gutachter und Mitglieder der Habilitationsgremien (Ausschüsse, Kommissionen usw.). Darüber hinaus kommen alle anderen Mitarbeiter der jeweiligen Fakultät, Vertreter anderer Universitätseinrichtungen usw. in Frage. Eine interessierte Öffentlichkeit kann letztendlich nicht ausgeschlossen werden. Eine Habilitationsordnung hat also einen öffentlichen, aber handlungsraumbezogenen Charakter - die meisten Adressaten in der recht heterogenen Rezipientengruppe stellen mit Sicherheit die im Hochschulwesen Agierenden dar. Der Adressatenkreis ist teilweise eingegrenzt. Was den Kommunikationskanal anbetrifft, handelt es sich bei einer Habilitationsordnung bekanntlich um eine schriftlich festgehaltene Textsorte, die in Druckform bekannt gegeben wird. In den letzten Jahren stellen die meisten Fakultäten ihre Ordnungen ins Netz, sie sind dann mit anderen Inhalten der Webpräsenz einer Fakultät verlinkt, so dass sie auch in Form eines mit dem gedruckten identischen elektronischen Dokuments (oft als pdf-Datei) erscheinen.

Ordnungen als Textsortenklasse erfreuen sich keines großen Interesses der (Rechts)Linguistik, sie finden in Typologien von juristischen Texten (z.B. J. Engberg 1997, D. Busse 2000) kaum Erwähnung. Ihnen wurden unseres Wissens auch keine linguistischen monographischen Einzelstudien gewidmet. Eine knappe, textlinguistische Beschreibung von Ordnungen findet man bei Ch. Fandrych/ M. Turmair (2011: 194ff.), die Ordnungen für Texte mit dem reglemetierend-handlungskontrollierenden Charakter halten. Ordnungen können normativen Texten zugeordnet werden, weil ihnen die kommunikative Funktion des verbindlichen Regelns in einem institutionellen Handlungsraum zugeschrieben wird. Es ist K. Brinker (2001: 124) Recht zu geben, dass sich die Textfunktion ("mit gewissen situativen und medialen Gegebenheiten") auf die Textstruktur auswirkt, und dementsprechend schlägt sich ihre dominierende regulierend-normative Funktion in der Struktur der Textsorte HO nieder. Es lassen sich zahlreiche Hinweise auf diese Textfunktionen in den Texten unseres Korpus finden, es sind dort primär sprachliche Ausdrücke des Verpflichtens (vgl. dazu B. Mikołajczyk/ J. Aptacy 2015a). Textstrukturell kann die HO als eine komplex aufgebaute Textsorte bezeichnet werden, die aus sehr unterschiedlichen Teilen (Abschnitten, Paragraphen usw.) besteht und über einen vergleichsweise großen Umfang verfügt. U.a. Verknüpfungssignale, die im Fokus dieses Beitrags stehen, weisen darauf hin, wie unterschiedliche Elemente des Textes, von Wörtern über Sätze und Absätze bis hin zu größeren Textteilen, mit anderen Elementen des Textes zusammenhängen. Die sprachliche Ausgestaltung ist in erster Linie durch einen recht homogenen fachlichen Wortschatz geprägt, es sind juristische Begriffe, die dem Bereich des Hochschulrechts entspringen (vgl. dazu B. Mikołajczyk/ J. Aptacy 2015b, B. Mikołajczyk/ J. Aptacy 2017 im Druck). 


\section{Textkohäsion}

Eine erfolgreiche Lektüre, d.h., das Verstehen eines Textes kann nur dann gewährleistet sein, wenn der Rezipient eine kohärente mentale Repräsentation eines im Text dargestellten Sachverhalts erzielt. In erster Linie kann er sich bei dieser anspruchsvollen kognitiven Aufgabe auf die Textkohäsion, stützen, d.h. auf formale Mittel in Texten, die auf Möglichkeiten der mentalen Verknüpfung von Textbestandteilen verweist.

Die Konstruktion einer kohärenten mentalen Repräsentation fordert kognitive Höchstleistungen von Rezipienten. Eine Erfolgsbedingung dafür ist, dass Texte ein zusammenhängendes Ganzes darstellen. (A. Schmitz 2016: 66)

Angesichts dessen bilden für P. Koch/ W. Oesterreicher (1994: 590) eine „durchstrukturierte semantische Progression und eine explizite Verkettung zwischen Sequenzen“ die Merkmale verständlicher Texte (vgl. auch A. Schmitz 2016: 68).

Im Folgenden kann aus Platzgründen nicht auf eine Diskussion der beiden für die Textlinguistik zentralen Begriffe Kohärenz und Kohäsion und ihre terminologischen Differenzierungen / ihre nicht einheitlich geregelte terminologische Abgrenzung eingegangen werden, einen aktuellen, überschaubaren und kritischen Überblick über die bisherige Betrachtung in der textlinguistischen Forschung liefert A. Schmitz (2016: 68ff.). Es gilt jedoch an dieser Stelle hervorzuheben, dass die Kohärenz aus der pragmatisch-funktionalen Perspektive als ein Mittel zur Ausführung einer bedeutungsvollen Handlung / eines bedeutungsvollen Sprechakts angesehen wird.

Wir verstehen unter Kohäsion in Anlehnung z.B. an A. Linke et al. (2004: 245) und M. Halliday/ R. Hasan (1976) sprachlich manifestierte Textbezüge, ergänzen aber diese nur auf sprachliche Phänomene beschränkte Charakteristik um andere (nicht sprachliche) im Text befindliche Mittel. Als Kohäsionsmittel werden demnach alle formalen Ausdrucksmöglichkeiten aufgefasst, die den Zusammenhalt des Textes bewirken und zur Kohärenz des Textes beitragen. Dies entspricht den pragmatisch orientierten textlinguistischen Ansätzen, in denen zwischen Kohäsion als Oberflächenphänomen und Kohärenz als den einem Text zugrundeliegenden, vom Textproduzenten zugrunde gelegten logischen Zusammenhängen, die nicht notwendigerweise an der Textoberfläche durch Kohäsionsmittel signalisiert sein müssen, unterschieden wird.

Schon bei R. de Beaugrande/ W.U. Dressler (1981) findet man eine grobe Einteilung der Kohäsionsmittel in die sog. lokale und globale Kohäsion. Die lokale Kohäsion erfasst benachbarte Textteile, verknüpft Sätze zu einem zusammenhängenden Textsegment. Es werden ausschließlich lokal begrenzte Beziehungen zusammengefasst. Dies wird bekanntlich z.B. mit Hilfe von Konnektoren, Rekurrenz, Proformen erreicht (Näheres zum Mittelinventar der lokalen Kohäsion vgl. T.A. Fritz 2005: 72ff.). Die globale Kohäsion zieht sich durch den ganzen Text hinüber und sorgt für den Zusammenhalt der Textsegmente. Sie umfasst die Verbindungen in den und zwischen den einzelnen Textsegmenten. Sie liegt vor, wenn Sätze oder Textteile, die im linearen Text weit auseinander liegen, als zusammengehörig erkannt werden. 


\section{Beschreibung des Belegmaterials}

In unserer Forschung analysieren wir ausgewählte HOs, die an Hochschulen in der Bundesrepublik Deutschland in Kraft sind. Den Ausgangspunkt bildete für uns die Web-Seite http:/www.hochschulen-deutschland.org/universitaeten.html (letzter Zugriff am 12.09.2016), auf der alle in Deutschland funktionierenden Hochschulen aufgelistet sind. Dazu gehören Universitäten, Fachhochschulen, Kunsthochschulen sowie Musikhochschulen. Laut der Landeshochschulgesetze besitzen nur Universitäten das Recht, Habilitationsverfahren durchzuführen, so dass sich unsere Forschung lediglich auf diesen Hochschultyp beschränkt. Auf der genannten Seite sind Informationen über die Verteilung der Universitäten auf einzelne Bundesländer zu finden, die folgendermaßen aussieht: Bayern - 13, Baden-Württemberg - 16, Berlin - 6, Brandenburg - 3, Bremen - 2, Hamburg - 4, Hessen - 7, Mecklenburg-Vorpommern - 2, Niedersachsen - 9, Nordrhein-Westfalen - 14, Rheinland-Pfalz - 6, Saarland - 1, Sachsen - 4, Sachsen-Anhalt - 2, Schleswig-Holstein -3 und Thüringen - 4. Insgesamt existieren also in Deutschland 96 Universitäten. Um die Repräsentativität des Korpus zu gewährleisten, haben wir beschlossen, von den Bundesländern, die mehr als neun Universitäten besitzen (Bayern, Baden-Württemberg, Nordrhein-Westfalen) jeweils vier auszuwählen, in den Ländern, in denen 6-9 Universitäten beheimatet sind (Berlin, Hessen, Niedersachsen, Rheinland-Pfalz), haben wir jeweils zwei berücksichtigt. Von den übrigen Bundesländern ( 3 und weniger Universitäten: Brandenburg, Bremen, Hamburg, Mecklenburg-Vorpommern, Saarland, Sachsen, Sachsen-Anhalt, Schleswig-Holstein und Thüringen) wurde jeweils eine Universität ausgewählt. Von den selegierten Universitäten haben wir jeweils eine HO untersucht, die an einer nach dem Zufallsprinzip ausgewählten Fakultät (bzw. im entsprechenden Fachbereich oder an der ganzen Universität) gilt. Zusätzlich wurden alle HOs an den angegebenen Universitäten durchgesehen.

Genauer analysiert wurden folgende HOs:

\begin{tabular}{|l|l|l|}
\hline HO_1 & U Passau & $\begin{array}{l}\text { Habilitationsordnung für die Juristische Fakultät der Universität Passau, } \\
\text { vom 23. Juli 2010 }\end{array}$ \\
\hline HO_2 & U München & $\begin{array}{l}\text { Habilitationsordnung für die Fakultät für Biologie der Ludwig-Maximi- } \\
\text { lians-Universität München vom 23. August 2004 }\end{array}$ \\
\hline HO_3 & TU München & $\begin{array}{l}\text { Habilitationsordnung der Technischen Universität München vom 9. De- } \\
\text { zember 2003 (in der Fassung der Änderungssatzung vom 13. Dezember } \\
\text { 2005) }\end{array}$ \\
\hline HO_4 & U Bamberg & $\begin{array}{l}\text { Habilitationsordnung für die Fakultäten Humanwissenschaften, Geistes- } \\
\text { und Kulturwissenschaften sowie Wirtschaftsinformatik und Angewandte } \\
\text { Informatik der Otto-Friedrich-Universität Bamberg, vom 20. September } \\
\text { 2007 }\end{array}$ \\
\hline HO_5 & U Heidelberg & $\begin{array}{l}\text { Habilitationsordnung der Universität Heidelberg für die Fakultät für } \\
\text { Physik und Astronomie, vom 3. Mai 2000 }\end{array}$ \\
\hline
\end{tabular}




\begin{tabular}{|c|c|c|}
\hline HO_6 & U Tübingen & $\begin{array}{l}\text { Habilitationsordnung der Universität Tübingen für die Wirtschaftswis- } \\
\text { senschaftliche Fakultät vom 22. September } 1997\end{array}$ \\
\hline HO_7 & U Mannheim & $\begin{array}{l}\text { Habilitationsordnung der Universität Mannheim vom 10. November } \\
2006\end{array}$ \\
\hline HO_8 & U Stuttgart & $\begin{array}{l}\text { Habilitationsordnung der Universität Stuttgart vom 6. September } 2006 \\
\text { (in der Fassung der Satzung zur Änderung der Habilitationsordnung der } \\
\text { Universität Stuttgart vom 1. März 2011) }\end{array}$ \\
\hline HO_9 & Humboldt U & $\begin{array}{l}\text { Habilitationsordnung der Theologischen Fakultät, veröffentlicht am } 4 . \\
\text { November } 2005\end{array}$ \\
\hline HO_10 & TU Berlin & $\begin{array}{l}\text {,Gemeinsame Kommission" Ordnung für die Habilitation in der Fakul- } \\
\text { tät Elektrotechnik und Informatik der Technischen Universität Berlin } \\
\text { vom 20. Dezember } 2002\end{array}$ \\
\hline HO_11 & U Viadrina & $\begin{array}{l}\text { Habilitationsordnung der Kulturwissenschaftlichen Fakultät der Europa- } \\
\text { Universität Viadrina Frankfurt (Oder) vom 25. Oktober } 1995\end{array}$ \\
\hline HO_12 & U Bremen & $\begin{array}{l}\text { Habilitationsordnung der Universität Bremen (Neufassung vom } \\
\text { 13.12.1999, } \\
\text { in der Fassung der letzten Änderungsordnung vom 11.7.2001 ) }\end{array}$ \\
\hline HO_13 & U Hamburg & $\begin{array}{l}\text { Habilitationsordnung des Fachbereichs Medizin der Universität Ham- } \\
\text { burg vom 7. Juli } 1999\end{array}$ \\
\hline HO_14 & U Frankfurt & $\begin{array}{l}\text { Habilitationsordnung des Fachbereichs Erziehungswissenschaften der } \\
\text { Johann Wolfgang Goethe-Universität Frankfurt am Main vom 12.Januar } \\
1993\end{array}$ \\
\hline \multirow[t]{2}{*}{ HO_15 } & U Gießen & $\begin{array}{l}\text { Rahmenbestimmungen für Habilitationsordnungen vom 16.6.1982/Än- } \\
\text { derung vom 21.12.1988 }\end{array}$ \\
\hline & & $\begin{array}{l}\text { Habilitationsordnung des Fachbereichs Humanmedizin der Justus-Lie- } \\
\text { big-Universität Gießen vom 01.02.1996 }\end{array}$ \\
\hline HO_16 & U Greifswald & $\begin{array}{l}\text { Habilitationsordnung der Ernst-Moritz-Arndt-Universität Greifswald } \\
\text { vom 18. Februar } 2004\end{array}$ \\
\hline HO_17 & U Hannover & Habilitationsordnung der Philosophischen Fakultät vom 16.3.2011 \\
\hline HO_18 & U Göttingen & $\begin{array}{l}\text { Habilitationsordnung der Georg-August-Universität Göttingen vom } \\
\text { 16.07.2008 }\end{array}$ \\
\hline HO_19 & U Bielefeld & $\begin{array}{l}\text { Habilitationsordnung der Fakultät für Psychologie und Sportwissen- } \\
\text { schaft der Universität Bielefeld vom 15. Januar } 2007\end{array}$ \\
\hline HO_20 & U Köln & $\begin{array}{l}\text { Habilitationsordnung der Medizinischen Fakultät der Universität zu } \\
\text { Köln vom 30.06.2010 }\end{array}$ \\
\hline HO_21 & TU Dortmund & Habilitationsordnung der Universität Dortmund vom 02.07.1993 \\
\hline
\end{tabular}




\begin{tabular}{|c|c|c|}
\hline HO_22 & BU Wuppertal & $\begin{array}{l}\text { Habilitationsordnung des Fachbereiches D - } \\
\text { Architektur, Bauingenieurwesen, Maschinenbau, Sicherheitstechnik } \\
\text { der Bergischen Universität Wuppertal vom 29. September } 2009\end{array}$ \\
\hline HO_23 & U Mainz & $\begin{array}{l}\text { Habilitationsordnung des Fachbereichs Physik, Mathematik und Infor- } \\
\text { matik der Johannes Gutenberg-Universität Mainz vom 23. Juli } 2007\end{array}$ \\
\hline HO_24 & U Trier & $\begin{array}{l}\text { Habilitationsordnung des Fachbereichs II: Sprach- und Literaturwissen- } \\
\text { schaften der Universität Trier vom 23. Juli } 1982\end{array}$ \\
\hline HO_25 & U Saarland & $\begin{array}{l}\text { Habilitationsordnung der Naturwissenschaftlich-Technischen Fakultäten } \\
\text { der Universität des Saarlandes. Vom 13. Februar } 2008\end{array}$ \\
\hline HO_26 & TU Dresden & Gemeinsame Habilitationsordnung der TU Dresden vom 14.02.1996 \\
\hline HO_27 & U Halle & $\begin{array}{l}\text { Habilitationsordnung der Wirtschaftswissenschaftlichen Fakultät der } \\
\text { Martin-Luther-Universität Halle-Wittenberg vom 22.06.94. geändert } \\
\text { durch die Satzung zur Änderung der Habilitationsordnung vom } \\
\text { 28.06.2000 }\end{array}$ \\
\hline HO_28 & U Kiel & $\begin{array}{l}\text { Habilitationsordnung (Satzung) der Technischen Fakultät der Christian- } \\
\text { Albrechts-Universität zu Kiel vom 01. Juni } 2006 \text { geändert durch } \\
\text { Satzung vom 29. Januar } 2010\end{array}$ \\
\hline HO_29 & TU Ilmenau & $\begin{array}{l}\text { Technische Universität Ilmenau Habilitationsordnung vom 01.07.2001 } \\
\text { geändert mit Schreiben vom 18.07.02 }\end{array}$ \\
\hline
\end{tabular}

\section{Datenanalyse}

In diesem linguistischen Beitrag wollen wir von der juristischen Klassifikation der Verweise (deklaratorische, konstitutive, normgenaue, inhaltsbezogene, starre, gleitende, Analogie-, Binnen- und Außenverweise, vgl. Handbuch der Rechtsförmlichkeit 2008, http://hdr.bmj.de/vorwort.html, letzter Zugriff am 16.02.2017) absehen und uns ausschließlich auf ihre formelle (sprachliche, graphische) Ausgestaltung und textuelle Leistung konzentrieren.

\subsection{Sprachliche Gestaltung und Funktion der Verweise}

$\mathrm{Zu}$ den wesentlichen Aspekten der Analyse der innertextuellen Verweise gehört neben der Beschreibung ihrer Form die Untersuchung ihrer Funktion, die ihren Gebrauch motiviert.

Einen ersten Schritt der Analyse stellt die Darstellung der sprachlichen Form der Verweise dar. Die im Untersuchungsmaterial ermittelten Verweise kommen als Phrasen und abhängige Sätze vor. Statistisch gesehen sind die Präpositionalphrasen dominierend. Seltener lassen sich Nominalphrasen und Quasi-Präpositionalphrasen feststellen. In diesem Beitrag werden nur Verweise in Form von Präpositionalphrasen thematisiert, alle anderen Formen, die statistisch gesehen in unserem Untersuchungskorpus im Vergleich zu Präpositionalphrasen äußert selten anzutreffen sind, werden nicht behandelt. 
Die Analyse setzt sich aus zwei Schritten zusammen, zuerst werden die Belege nach der Form klassifiziert und charakterisiert. Danach erfolgt eine syntaktische Beschreibung. Die Reihenfolge der präsentierten Phänomene richtet sich nach der Häufigkeit ihres Auftretens.

\subsection{1. gemäß-Phrasen}

\begin{tabular}{|l|l|l|l|l|l|l|}
\hline 1 & gemäß/gem. & $\S$ & & & & 42 \\
\hline $1 \mathrm{a}$ & gemäß & $\S \S / \S$ und $\S$ & & & & 3 \\
\hline 2 & gemäß/gem. & $\S$ & Absatz/Abs. & & & 48 \\
\hline 3a & gemäß/gem. & $\S$ & Absatz/Abs. & Satz & & 13 \\
\hline 3b & gemäß/gem. & $\S$ & Absatz/Abs. & Ziffer/Ziff. & & 14 \\
\hline 4aı & gemäß & $\S$ & Absatz & Satz & Ziffer & 4 \\
\hline $4 \mathrm{a} 2$ & gemäß & $\S$ & Absatz & Ziffer & Satz & 4 \\
\hline 4b & gemäß & $\S$ & Absatz & Ziffer & Buchstabe & 3 \\
\hline 4c & gemäß & $\S$ & Absatz/Abs. & & Nr. & 12 \\
\hline 5 & gemäß/gem. & & Absatz/Abs. & & & 26 \\
\hline 6a & gemäß/gem. & & Absatz/Abs. & Satz & & 3 \\
\hline 6b & gemäß & & Absatz & Ziffer & & 3 \\
\hline 7 & gemäß & & & Satz & & 2 \\
\hline 8a & gemäß & & & & Nr. & 4 \\
\hline 8b & gemäß & & & & Buchstabe & 2 \\
\hline 8c & gemäß & & & $\begin{array}{l}\text { Muslage/dem in An- } \\
\text { lage }\end{array}$ & 2 \\
\hline & & & & & 185 \\
\hline
\end{tabular}

Die obige Tabelle präsentiert die Vorkommenshäufigkeit der gemäß-PPs im untersuchten Korpus, wobei für die Aussonderung der einzelnen (Sub)Typen der Aufbau dieser Phrasen als Unterscheidungskriterium gilt. Mit Aufbau ist hier die explizite Bezugnahme auf Gliederungseinheiten eines juristischen Textes, in diesem Fall einer HO, gemeint. Es geht somit darum, ob gemä $\beta$ z.B. die NP $\xi(\xi)$ als Komplement zu sich nimmt oder ob das Komplement um Exponenten kleinerer Gliederungseinheiten wie 
Abs./Absatz, Buchstabe, Nr./Nummer etc. appositiv erweitert ist. Die Tabelle zeigt auch auf überschaubare Weise, in welchen Kombinationen und mit welcher Häufigkeit die Namen und Symbole der genannten Einheiten vorkommen.

Folgende syntaktische Funktionen der gemä $\beta$-Phrasen lassen sich in den untersuchten Texten feststellen. Die Reihenfolge entspricht der Häufigkeit ihres Auftretens:

- Attribut

Gemäß-Phrasen als Attribute 1. und 2. Grades beziehen sich unterschiedlich auf übergeordnete Nomina und sind ihnen in der Regel nachgestellt. Dabei sind Attribute 1. Grades am häufigsten und abgesehen von einem Fall, in dem die gemä $\beta$-Phrase auf ein substantivisch gebrauchtes Partizip rekurriert und aus diesem Grunde ihm vorangestellt ist, bilden sie sich auf ein Substantiv beziehende und nach ihm stehende Attribute. Sie kommen insgesamt 96 mal vor, wie z.B.

(1) Prüfungsvergünstigungen gemäß Abs. 2 werden nur auf schriftlichen Antrag gewährt. (HO_1)

(2) Das Recht, sich an der Diskussion zu beteiligen, haben alle gemäß Abs. 2 Sätze 2 und 3 persönlich Eingeladenen. (HO_10)

An zweiter Stelle treten Attribute 2. Grades (52 mal) auf. Sie vertreten alle denselben Typ, d.h., sie erscheinen als nachgestelltes Attribut zu einem Genitiv- oder Präpositionalattribut.

(3) Die Urkunde trägt das Datum der Beschlussfassung gemäß § 12 Abs. 4. (HO_3)

Viel seltener werden Attribute 3. Grades (8 mal) eingesetzt:

(4) Eine Wiederholung eines gem. Abs. 3 Satz 3 gescheiterten Habilitationsverfahrens ist ausgeschlossen. (HO_3)

(5) Der Termin für die Wiederholung der Leistung gem. Abs. 1 sollte spätestens 6 Wochen nach der Erbringung des ersten wissenschaftlichen Vortrages/des ersten Lehrvortrages liegen. (HO_25)

Hier kann man zwei Strukturtypen unterscheiden, einerseits nachgestelltes Attribut zu einem Genitivattribut, andererseits ein vorangestelltes, das auf das erweiterte Attribut konstituierende Partizip Bezug nimmt.

Eine Klassifizierung der Attribute nach ihrem Abhängigkeitsgrad wird u.a. deswegen durchgeführt, weil die syntaktische Struktur sich auf ihre semantische Interpretation auswirkt und somit das Textverständnis mit determiniert. Vielfach lassen sich in isolierten Sätzen die Attribuierungsverhältnisse nur schwer ermitteln, d.h., es muss auf den Gesamttext zurückgegriffen werden, um feststellen zu können, auf welche Größe sich das Attribut bezieht. Im unten stehenden Beleg kann die gemäß-PP z.B. auf Vorschlag, Zusammensetzung und Habilitationskommission Bezug nehmen, sie kann sogar als Adverbial das Verb erstellen modifizieren. Erst nach der Lektüre des entsprechenden Absatzes konnte erschlossen werden, dass die gemäß-PP das Nomen Zusammensetzung modifiziert und somit Attribut 2. Grades ist:

(6) Nach Eingang des Antrags auf Annahme als Habilitand/Habilitandin setzt das Dekanat das Fachmentorat ein und erstellt einen Vorschlag für die Zusammensetzung der Habilitationskommission für den Fakultätsrat gemäß §1 Abs. 5. (HO_25) 


\section{- Adverbial}

Neben der syntaktischen Rolle eines Attributs übernimmt die gemäß-PP die Funktion eines modalen Adverbials (17 mal belegt). Da nimmt sie auf das Prädikat Bezug:

(7) Entsprechen die Ergebnisse den Vorgaben der Zielvereinbarung, wird das Habilitationsverfahren gemäß § 13 fortgeführt. (HO_1)

Mitunter begegnen Beispiele, an deren Struktur nicht abzulesen ist, ob die gemäßPP als Attribut oder Adverbial fungiert, z.B. in:

(8) Wird die Annahme einer Habilitationsleistung gemäß Satz 1 zurückgenommen, so gilt das Habilitationsverfahren als erfolglos beendet. (HO_8)

Hier konnte erst nach der Lektüre der genannten Stelle eine eindeutige Zuordnung als Adverbial vorgenommen werden. In Satz 1 wird nämlich geschildert, unter welchen Bedingungen die Annahme der Habilitationsleistung zurückgenommen wird.

- Syntaktisch nicht integriert, ambige Lesart zwischen Attribut und Adverbial

Vereinzelt (2 Belege) begegnet eine syntaktisch nicht integrierte Verwendung der betreffenden Wortgruppe. Es geschieht durch die Einklammerung, z.B.:

(9) Im Anschluß an die Aussprache entscheidet der erweiterte Fakultätsrat mit der Mehrheit der Professoren und habilitierten Mitglieder (gemäß § 3 Abs. 5), ob der Bewerber den Probevortrag und die wissenschaftliche Aussprache bestanden hat. (HO_11)

Festzuhalten ist, dass die gemäß-Phrase in den HOs für die Bildung eines Attributs prädestiniert ist. Als eine viel seltenere Funktion gilt das Adverbial.

Es lassen sich folgende Eigenschaften von gemäß ermitteln, die ihnen ungeachtet ihrer syntaktischen Funktion und ihrer Abhängigkeitsstruktur gemeinsam sind, und zwar sind das die Position und Rektion der Präposition in der Phrase. Laut z.B. der DUDEN-Grammatik (2009: 602f.) schwankt gemäß zwischen der Prä- und Postposition in der Phrase, die sie regiert. Dies bestätigt Grammis des IdS in Mannheim ${ }^{1}$.

Gemäß der Anweisung des Chefs wurde der unpünktliche Mitarbeiter entlassen. ${ }^{2}$

Der Anweisung des Chefs gemäß wurde der unpünktliche Mitarbeiter entlassen.

Diese Aussagen treffen auf das untersuchte Sprachmaterial nicht zu. Die einzige Stellung bei 185 gefundenen Phrasen ist die Ante- oder Präposition. Ein weiteres Merkmal von gemäß, das in gegenwärtigen grammatischen Arbeiten diskutiert wird, ist seine Rektion. Auch hier sind sich die Duden- und IdS-Autoren einig, gemäß regiere nämlich sowohl den Dativ als auch den Genitiv. Nach Duden. Richtiges und gutes Deutsch (2001: 348) jedoch "[verlangt] [d]ie Präposition gemäß [...] den Dativ (nicht den Genitiv)". Nach der Duden-Grammatik (2006: 618) kommt bei gemäß gelegentlich die Genitivrektion vor, die stilistische Gründe haben dürfte. Der Genitiv ist jünger und

\footnotetext{
${ }^{1}$ http://hypermedia.ids-mannheim.de/call/public/gramwb.ansicht?v_ $\mathrm{app}=\& v_{\text {_k }}$ kat $=$ gramm\&v_buchstabe $=$ G\&v_id=2476, letzter Zugriff am 13.11.2016 ${ }^{2}$ Beispiele nach http://www.deutschegrammatik20.de/ praepositionen/die-bedeutung-der-prapositionen-ubersicht/die-bedeutung-der-prapositionengemas/, letzter Zugriff am 13.11.2016.
} 
kommt v.a. in offiziellen geschriebenen Texten vor, stilistisch wirkt er als höher stehend (vgl. auch C. Di Meola 2004: 166). Man sieht hier also, dass die Meinungen der Linguisten bezüglich der gemä $\beta$-Rektion auseinandergehen.

Eine genaue Betrachtung des empirischen Materials lässt folgende Aussagen zu:

- die in der gemäß-Phrase auftretenden Substantive werden in Bezug auf ihre aktuelle Kasusform nicht markiert, so dass eine eindeutige Zuordnung nicht möglich ist. Die Autoren der Habilitationsordnungen meiden den Gebrauch von Artikelwörtern, auch die Kasussuffixe finden keine Verwendung. Dort, wo in anderen Rechtstexten z.B. die Pluralform mit dem entsprechenden Artikel gekennzeichnet ist, (vgl. Die zuständige Behörde übermittelt die Daten gemäß den Absätzen 1 bis 4 dem Deutschen Institut für medizinische Dokumentation.... Gesetz über Medizinprodukte ${ }^{3}$, fehlt eine solche Markierung in den untersuchten HOs durchgehend. Nur in zwei Fällen wird der Kasus als Dativ kenntlich gemacht. Dies entspricht also der linguistischen Beschreibung:

(10) Dazu reicht der Habilitand/die Habilitandin dem Dekanat unaufgefordert einen Zwischenbericht gegliedert gemäß dem Muster in Anlage 2 zu dieser Ordnung ein, der die bis dahin erbrachten Leistungen gemäß § 5 Abs. 4 enthält. (HO_25)

- Zum anderen erscheint der appositive Nominativ (vgl. dazu H. Vater 2007: 28ff.), der in solchen Strukturen sich immer mehr durchsetzt:

(11) eine Erklärung der Bewerberin oder des Bewerbers, dass es sich dabei um eigene wissenschaftliche Leistungen handelt (gegebenenfalls eine Darstellung gemäß § 3 Absatz 1 letzter Satz), (HO_23)

\subsection{2. nach-Phrasen}

Die zweithäufigste Präposition, die in PPs mit intratextueller Verweisfunktion vorkommt, ist nach. Die Semantik von nach ist der von gemäß, entsprechend, zufolge ähnlich (vgl. Duden. Deutsches Universalwörterbuch 2001: 627). Die Präposition wird bei "Entsprechung, Übereinstimmung. Sinngemäße[r] Wiedergabe mit der Möglichkeit der Distanzierung" verwendet (G. Helbig / J. Buscha 1994: 434). Die polyseme Präposition $n a c h^{4}$ besitzt in einer ihrer Bedeutungsvarianten eine der Präposition ge$m \ddot{a} \beta$ ähnliche Bedeutung und bezeichnet das Muster, Maß, Vorbild oder Modell, nach dem sich eine bestimmte Person oder Sache richten soll (vgl. H. Weinrich 1993: 658; Duden. Deutsches Universalwörterbuch 2001: 1113). Die Präposition gemäß dient der Redewiedergabe und benennt die Quelle einer Aussage. Die Bedeutung von gemä $\beta$ entspricht der Bedeutung der Präposition $n a c h^{5}$. Die untersuchten Texte scheinen diese definitorischen Merkmale zu bestätigen, d.h., es wird auf Inhalte in Textstellen verwiesen, nach denen man sich als Akteur des Habilitationsverfahrens bei der Ausführung einer Handlung sinngemäß richten soll.

\footnotetext{
${ }^{3}$ https://www.gesetze-im-internet.de/mpg/_25.html, letzter Zugriff am 13.11.2016.

${ }^{4}$ Duden. Deutsches Universalwörterbuch (2001: 1113) führt sieben verschiedene Bedeutungsvarianten (mit mehreren Untervarianten) auf.

5 Vgl. http://www.deutschegrammatik20.de/praepositionen/die-bedeutung-der-prapositionenubersicht/die-bedeutung-der-prapositionen-gemas/, letzter Zugriff am 17.11.2016.
} 


\begin{tabular}{|c|c|c|c|c|c|c|c|}
\hline 1. & nach & $\S$ & & & & & 36 \\
\hline $1 \mathrm{a}$ & nach & $\S \S /$ Paragraphen & & & & & 9 \\
\hline 2. & nach & $\S$ & Absatz / Abs. & & & & 35 \\
\hline 3.a & nach & $\S$ & Absatz / Abs. & Satz & & & 10 \\
\hline $3 . b$ & nach & $\S$ & Absatz / Abs. & Ziffer / Ziff. & & & 7 \\
\hline 4. & nach & $\S$ & Abs. & Satz & Buchst. & & 1 \\
\hline 5. & & $\S$ & Absatz & & & Nr. & 1 \\
\hline $6 . \mathrm{a}$ & nach & $\S$ & & Satz & & & 3 \\
\hline $6 . b$ & nach & $\S$ & & Ziffer/Ziff. & & & 6 \\
\hline 7 & nach & $\S$ & & Satz & Buchst. & & 1 \\
\hline 8. & nach & & Absatz / Abs. & & & & 39 \\
\hline $9 . \mathrm{a}$ & nach & & Absatz / Abs. & Satz & & & 6 \\
\hline $9 . b$ & nach & & Absatz & Ziffer / Ziff. & & & 2 \\
\hline 10 & nach & & Absatz & Satz & Nr. & & 1 \\
\hline 11 & nach & & Absatz & & Nr. & & 1 \\
\hline 12.a & nach & & & Satz & & & 7 \\
\hline 12.b & nach & & & Ziffer & & & 1 \\
\hline 13. & nach & & & & Nr. & & 3 \\
\hline 14 & nach & & & & $\begin{array}{l}\text { Maßgabe } \\
\text { dieser } \\
\text { Ordnung / } \\
\text { den Vor- } \\
\text { schriften }\end{array}$ & & $1 / 1$ \\
\hline & & & & & & & 171 \\
\hline
\end{tabular}

Der obigen Tabelle ist zu entnehmen, dass nach ähnlich wie gemäß auf verschiedene Gliederungseinheiten der HOs verweisen kann. Es sind also Paragraphen, Absätze, Nummern etc. in verschiedenen Kombinationen, auf die mit nach verwiesen wird. Von den syntaktischen Funktionen können die PPs folgende erfüllen: 


\section{- Attribut}

Innerhalb dieser syntaktischen Funktion können unterschiedliche Grade des Bezugs festgestellt werden. Beim Attribut ersten Grades, das in unserem Untersuchungsmaterial 87 mal auftritt, handelt es sich um die frequentativste Verwendung:

(12) Ist der Antrag nach $§ \mathbf{6}$ unvollständig, setzt der Dekan oder die Dekanin dem Bewerber oder der Bewerberin eine angemessene Frist zur Vervollständigung. (HO_1)

Dem folgt in der Häufigkeit des Vorkommens das Attribut zweiten Grades, 54 mal registriert:

(13) Insbesondere ist ihnen, wenn die Art der Behinderung es rechtfertigt, eine Verlängerung der Fristen nach § 9 Satz 1 und $§ 12$ Abs. 1 um bis zu einem Viertel zu gewähren. (HO_1)

Vereinzelt stößt man auf Attribute dritten Grades, die in den HOs 14 mal festgestellt wurden:

(14) Ist außerhalb der Fakultät schon ein Habilitationsverfahren für das in $\S 5$ Abs. 1 Satz 2 bezeichnete Fach oder Fachgebiet erfolglos beendet worden, gilt die Zulassung als Zulassung zur Wiederholung des Verfahrens nach § 11. (HO_6)

(15) Die nach Absatz 4 erfolgte Bestellung der Berichterstatter bleibt aufrechterhalten, wenn keine andere Entscheidung getroffen wird. (HO_6)

(16) Nach dem Beschluß über die Annahme der schriftlichen Habilitationsleistung nach $\S$ 8 Abs. 7 Satz 1 entscheidet der Habilitationsausschuß über Themenvorschläge des Bewerbers für den von ihm nach Absatz 3 zu haltenden wissenschaftlichen Vortrag. (HO_6)

Seltener sind Attribute höheren Grades als drei festzustellen, dennoch treten vereinzelt auch Attribute vierten (zweimal) Grades:

(17) schließlich eine Versicherung über die Vollständigkeit des Verzeichnisses der wissenschaftlichen Veröffentlichungen nach Ziffer 4, (HO_6)

(18) Die Annahme von Habilitationsleistungen bedarf einer Zweidrittelmehrheit der anwesenden Mitglieder des erweiterten Fakultätsrates mit Stimmrecht nach Abs. 2 (HO_11)

und fünften (einmal) Grades auf:

(19) das Datum des Beschlusses des Fakultätsrates über den erfolgreichen Abschluss des Habilitationsverfahrens nach Absatz 1, (HO_26)

Abgesehen von ein paar Fällen, in denen die nach-Phrase als Bestandteil eines erweiterten Attributs (3. Grades) zu finden ist, wird sie durchgehend als ein postpositioniertes Präpositionalattribut verwendet.

Wie dargelegt, dominiert bei den nach-PPs die attributive Funktion, aber es wird auch die adverbiale realisiert (neunmal):

(20) Im Fall der Aussetzung des Verfahrens ist nach Ablauf der gesetzten Frist erneut nach Absatz 4 bis 6 zu verfahren. (HO_6) 
(21) An den Entscheidungen im Habilitationsverfahren wirkt nach Maßgabe dieser Ordnung der erweiterte Fakultätsrat gemäß $\S 90$ Abs. 3 des Brandenburgischen Hochschulgesetzes mit. (HO_11)

Ähnlich wie bei den gemäß-PPs wurden syntaktisch ambige Phrasen festgestellt, die ohne Kontext eine Alternative zwischen der attributiven und adverbialen Lesart zulassen, jedoch wird diese Doppeldeutigkeit disambiguiert, sobald man sich mit dem entsprechenden Textpassus bekannt gemacht hat. Im untenstehenden Beispiel hat man es mit der adverbialen Funktion zu tun:

(22) Sobald in einem Fall des Absatzes 6 der Fakultätsrat der im Schwerpunkt zuständigen Fakultät die Arbeit nach $\S 7$ Abs. 4 angenommen hat, bestellt der Fakultätsrat der anderen Fakultät zwei Gutachter. (HO_16)

Obwohl bezüglich der Rektion der Präposition nach in der normativen Literatur keine Meinungsverschiedenheiten bestehen ${ }^{6}$, so dass unzweifelhaft von der Dativrektion auszugehen ist, werden die Kasusexponenten fast nie sichtbar gemacht ${ }^{7}$. Lediglich in vereinzelten Fällen (genau vier) ist an Determinatoren (und Flexionsendungen) der Dativ Plural abzulesen, vgl.:

(23) das aufgenommene Verfahren gelten die Bestimmungen über das Verfahren nach den Paragraphen 7 und 8 mit der Maßgabe, dass eine erneute Aussetzung des Verfahrens ausgeschlossen ist. (HO_25)

(24) Das bei dieser Feststellung anzuwendende Verfahren richtet sich nach den $§ \S 8$ bis 10. (HO_6)

Während in der Regel mit nach auf juristische Textgliederungseinheiten vom Typ $\S(\xi), A b s$. etc. verwiesen wird, haben wir es bei dem unten stehenden Beleg mit einer expliziten Bezugnahme auf den Inhalt des Absatzes, was aus unserer Sicht eine Redundanzerscheinung darstellt, da die Absätze in unserem Korpus ausschließlich Vorschriften enthalten.

(25) Kommen die Gutachten zu stark voneinander abweichenden Ergebnissen und sieht sich der 'erweiterte Fakultätsrat deshalb zu einem abschließenden Vorschlag für die Beurteilung der schriftlichen Habilitationsleistung nicht in der Lage, so werden weitere Gutachter nach den Vorschriften des Absatzes 2 bestellt. (HO_11)

\subsubsection{Syntaktische Eigenschaften von gemäß und nach in Gegenüberstellung}

Die folgende Tabelle gibt einen Überblick über die sich aus der Analyse unserer Sprachdaten ergebenden Charakteristika beider Präpositionen an, deren Gebrauch als eine für die unter die Lupe genommene Textsorte typische Markierung der Verweise einzustufen ist. Die abweichenden Merkmale werden mit Grau unterlegt. Der Tabelle

\footnotetext{
${ }^{6}$ Vgl. aber C. Di Meola (2004: 172): nach des Umbaus ('after the renovation'/SGT, November 30, 1998), die Frage nach des künstlerischen Dialogs ('the question of the artistic dialogue'/ MM, January 26, 1998).

7 „Unterlassene Deklination” (A. Paulfranz 2013).
} 
kann entnommen werden, dass zwischen den Präpositionen kaum syntaktische Unterschiede festgestellt wurden. Die oben zitierten Wörterbücher machen auch auf keine bzw. lediglich auf geringfügige Unterschiede der Semantik der beiden Präpositionen (innerhalb der in dieser Textsorte verwendeten Lesart - Angabe der Quelle) aufmerksam. Es wird bei der Untersuchung der Textebene versucht herauszufinden, ob sich signifikante funktionale Unterschiede bei der verweisenden Verwendung gemäß und nach beobachten lassen.

\begin{tabular}{|c|c|c|c|}
\hline & & gemäß-PP & nach-PP \\
\hline \multirow[t]{3}{*}{ syntaktische Funktion } & Attribut & + & + \\
\hline & Adverbial & + & + \\
\hline & syntaktisch nicht integriert & + & - \\
\hline \multirow[t]{2}{*}{ Position des Attributs } & Postposition & + & + \\
\hline & Anteposition (im erweiterten Attribut) & + & + \\
\hline Rektion der Präposition & Kasusmarkierung & - & - \\
\hline
\end{tabular}

\subsubsection{Quasi-Präpositionalphrase (im Sinne/ im Falle)}

\begin{tabular}{|l|l|l|l|l|l|l|l|}
\hline I.1 & im Sinne & des $($ der $)$ & $\S(\S \S)$ & & & & $4(1)$ \\
\hline I.2 & im Sinne & des & $\S$ & Abs. & & & 1 \\
\hline I.3a & im Sinne & des & $\S$ & Abs. & Satz & & 1 \\
\hline I.3b & im Sinne & des & $\S$ & Abs. & & Nr. & 2 \\
\hline I.3c & im Sinne & des & $\S$ & Abs. & & Ziffer & 1 \\
\hline I.4 & im Sinne & des & & Abs. & & & 3 \\
\hline I.5 & im Sinne & des & & Abs. & & Nr. & 1 \\
\hline II.1 & im Sinne & von & $\S$ & Absatz & & & 1 \\
\hline
\end{tabular}




\begin{tabular}{|l|l|l|l|l|l|l|l|}
\hline II.2 & im Sinne & von & & Abs. & & & 2 \\
\hline II.3 & im Sinne & von & & Absatz & & Nr. & 2 \\
\hline II.4 & im Sinne & von & & Abs. & Satz & Nr. & 2 \\
\hline II.5 & im Sinne & von & & & Satz & & 1 \\
\hline III.1 & i.S. & des & $\S$ & Abs. & & & 1 \\
\hline III.2 & i.S. & d. & $\S$ & Abs. & Satz & & 1 \\
\hline & & & & & & & 23 \\
\hline
\end{tabular}

Aus der obigen Tabelle ist zu ersehen, dass im Sinne im Vergleich zu gemäß und nach als intratextueller Verweisexponent relativ selten verwendet wird, dennoch ist seine Vorkommenshäufigkeit beachtenswert. Bevor jedoch seine Funktionen im untersuchten Korpus einer Analyse unterzogen werden, soll zunächst sein kategorialer Status kurz diskutiert werden.

Äußerlich gesehen hat dieser Verweis die Form einer PP vom Typ Präp + Det + N, wobei die Präposition mit dem Artikel zu einem Portemanteau-Morphem kontrahiert ist. Nun ist zu überlegen, ob sich dieser Ausdruck als eine prototypische, nicht lexikalisierte PP klassifizieren lässt oder ob er sich möglicherweise als ,präpositionsartige Präpositionalphrase“ (vgl. U. Engel 1988: 699ff.) auf dem Entwicklungsweg zu einer Präposition befindet. Nach der Duden-Grammatik (2006: 607) werden (i) einfache (in, auf, mit ..) und (ii) komplexe Präpositionen (mithilfe, zufolge, anhand, anstelle ..) sowie (iii) präpositionsartige Wortverbindungen (im Verlauf(e) (von), in Bezug auf, in Anbetracht, an Stelle ..) unterschieden. ${ }^{8}$ Am Beispiel von anstelle aus (ii) und an Stelle aus (iii) kann gesehen werden, dass die Übergänge zwischen den einzelnen Klassen fließend sein können und dass die Entstehung der prototypischen, d.h. einfachen Präpositionen über die Stufen (ii) und (iii) erfolgen kann. Insbesondere ist die Verbindung von Präp und N eine häufige Quelle neuer Präpositionen (vgl. C. Di Meola 2004: 168; Duden-Grammatik 2006: 607f.). Eine ähnliche Klassifizierung bieten auch G. Helbig/ J. Buscha (1991: 402ff.), die primäre und sekundäre Präpositionen unterscheiden. Die ersteren wie an, auf, durch, neben etc. bilden eine relativ geschlossene Klasse und in der Gegenwartssprache lassen sie sich nicht als polymorphematische Wortbildungskonstruktionen erkennen. Zu den sekundären Präpositionen rechnen die Autoren Ableitungen von Wörtern anderer Wortklassen (z.B. betreffs, gelegentlich, zuzüglich) sowie Zusammensetzungen und Wortgruppen aus Präp + N (z.B. anhand, auf Grund /

${ }^{8}$ C. Di Meola (1999: 347) spricht von stark grammatikalisierten und schwach grammatikalisierten Adpositionen. Dies würde im ersten Fall der Klasse (i) und im zweiten den Klassen (ii) und (iii) der Duden-Grammatik entsprechen. Die stark grammatikalisierten Adpositionen werden ausschließlich als grammatische Funktionswörter verwendet, während die schwach grammatikalisierten auch als Inhaltswörter fungieren können. 
aufgrund, im Laufe). Die meisten sekundären Präpositionen regieren den Genitiv bzw. in Ersatzfunktion den Dativ mit von. Eine Sondergruppe bilden Verbindungen, in denen das Kernnomen eine weitere Präposition regiert wie in Bezug auf, in Verbindung mit oder im Gegensatz zu. Alle sekundären Präpositionen kommen nicht in Objekten vor, sondern in Attributen und Adverbialbestimmungen, wobei sie ihre lexikalische Bedeutung bewahren (vgl. G. Helbig/ J. Buscha 1991: 403).

Für den Status von im Sinne als grammatikalisierter „Quasipräposition“ / uneigentlicher Präposition / präpositionsartiger Wortverbindung (Duden-Grammatik 2006: 607) / präpositionsartiger Präpositionalphrase (U. Engel 1988: 699) in den untersuchten Texten sprechen u.E. folgende Merkmale (vgl. auch G. Helbig/ J. Buscha 1991: 403f.): ${ }^{9}$

- Unveränderliche (archaische) Form in der hier realisierten Funktion: *im Sinn, *in einem Sinn(e), *in dem Sinne, *im guten Sinne u.Ä.. Dies unterscheidet diesen Ausdruck vom homophonen im Sinne wie in Sie hat in meinem Sinne gehandelt, etwas im Sinne haben oder idiomatisch jdm. nicht in den Sinn wollen. $^{10}$

- Feste Rektion: Genitiv oder von-PP als ,analytischer“ Genitiv,

- Verblasste Bedeutung des Autosemantikums, die durch solche primären Präpositionen wie nach oder gemäß wiedergegeben werden könnte.

\subsection{5. im Falle /in einem Fall / in (den) Fällen}

\begin{tabular}{|l|l|l|l|l|l|l|}
\hline I.1 & im Falle (in einem Fall) & des & Absatzes & & & $2(1)$ \\
\hline I. 2 & im Falle & des & & Satzes & & 2 \\
\hline II.1 & in den Fällen & des & $\S$ & Absatz & & 1 \\
\hline II.2 & in den Fällen & der & & Absätze & & 1 \\
\hline II.3 & in den Fällen & der/des & & $\begin{array}{l}\text { Absätze/ } \\
\text { Absatzes }\end{array}$ & Nr./Nrn & 2 \\
\hline II.4 & in Fällen & von & & Absatz & & 1 \\
\hline
\end{tabular}

Ein zum Teil der Verbindung im Sinne ähnliches Verhalten weist die PP im Falle / in den Fällen auf. Auch sie verweist auf andere Stellen im Text (Absatz und Satz) und

\footnotetext{
${ }^{9}$ Allerdings findet man im Sinne als uneigentliche Präposition weder in der Duden-Grammatik (2006) noch in C. Di Meola (1999; 2004), lediglich in U. Engel (1988: 701) erscheint es als präpositionsartige Präpositionalphrase (,Hanne hat das im Sinne des Gründers der Firma geregelt.").

${ }^{10}$ G. Helbig/ J. Buscha (1991: 403) bringen ein Beispiel, in dem das Kernnomen nur als Bestandteil einer sekundären Präposition vorkommt: in Anbetracht vs. *der Anbetracht.
} 
zeichnet sich durch eine feste (analytische) Genitivrektion aus. Sie ist aber nicht formkonstant, erscheint in den analysierten HOs in drei Ausprägungen: mal im Singular im Fall, in einem Fall und einmal im Plural in den Fällen. Im Vergleich zu im Sinne fällt demnach auf, dass hier eine bestimmte Formvariabilität vorliegt, was die Vermutung nahelegt, dass solche Ausdrücke einen geringeren Grammatikalitätsgrad aufweisen als im Sinne. Der Ausdruck hat den Grammatikalisierungsprozess noch nicht durchlaufen und erfüllt von den oben genannten Kriterien nur das der festen Rektion. Die beiden PPs (im Sinne und mit im Falle mit all seinen Varianten) haben die verweisende Funktion gemeinsam.

Des Weiteren werden in der verweisenden Funktion innerhalb einer Habilitationsordnung weitere PPs (Entscheidung zu, Maßgabe von), auch NPs (Genitivphrasen, Nominativphrasen, Appositionen) und Nebensätze eingesetzt. Ihre Verwendung ist jedoch im Vergleich zu den oben diskutierten Mitteln marginal. Aus diesem Grunde werden sie in unseren Ausführungen nicht thematisiert.

\subsection{Textuelle Leistung}

\subsubsection{Mikro- und makrostrukturelle Verweise}

Eine erste grobe Unterteilung der bezugnehmenden Elemente kann nach ihrem Geltungsbereich im Text vorgenommen werden. Nach diesem textuell-räumlichen Kriterium kann man zwei Arten von Verweisungen aussondern:

1. Diejenigen, die innerhalb einer Texteinheit, z.B. eines Paragraphen operieren:

\section{(26) §8 Habilitationskommission}

(1) Der gemäß $\S 7$ zu bestellenden Habilitationskommission gehören an:

1. zwei der Gutachter bzw. Gutachterinnen gemäß $§ 7$ Absatz 2, darunter mindestens ein Professor bzw. eine Professorin der Universität Bremen;

2. zwei weitere Professoren, Professorinnen oder Habilitierte, die das Lehr- und Forschungsgebiet vertreten, für das die Habilitation angestrebt wird, darunter mindestens ein Mitglied der Universität Bremen, das nicht der Gruppe der Professoren und Professorinnen angehören muss, wenn beide Gutachter bzw. Gutachterinnen Professoren bzw. Professorinnen der Universität sind;

3. als weitere Mitglieder, die an der Arbeit der Kommission mit beratender Stimme teilnehmen:

a) eine akademische Mitarbeiterin oder ein akademischer Mitarbeiter,

b) eine sonstige Mitarbeiterin oder ein sonstiger Mitarbeiter,

c) zwei Studierende.

Verzichtet die Gruppe der sonstigen Mitarbeiterinnen und Mitarbeiter im Fachbereichsrat auf die Besetzung des Mandats gemäß Buchstabe b, entsendet die Gruppe der akademischen Mitarbeiter und Mitarbeiterinnen zwei Vertreter bzw. Vertreterinnen in die Kommission.

Die Mitglieder gemäß Nr. 3 müssen Mitglieder der Universität Bremen sein. Das Mitglied gemäß Nr. 3a muss in dem Fach, in dem die Habilitation angestrebt wird, wissenschaftlich arbeiten. Die Mitglieder gemäß Nr. 3c müssen das Fach studieren. (HO_12)

Sie sind als ein Ausdruck der lokalen Kohäsion der untersuchten Texte aufzufassen, weil sie auf der mikrostrukturellen Textebene ihre Verbindungskraft zeigen und 
dementsprechend werden sie mikrostrukturelle Kohäsionsmittel / mikrostrukturelle (= lokale) Verweise genannt. Verweise, die Verknüpfungen innerhalb eines $\mathrm{Pa}$ ragraphen herstellen, kommen nur in strukturierten Paragraphen vor, die aus mehr als zwei Struktureinheiten bestehen, es können Absätze, seltener Punkte sein, die als Elemente der Konnexion in Frage kommen. Mikrostrukturelle Verweise operieren in zwei Richtungen, wie in Beleg (HO_12) kataphorisch, seltener anzutreffen ist die anaphorische Funktion.

2. Solche, die über die Grenzen eines Paragraphen hinausgehen:

(27) § 6 Abs. 2 Bei Erfüllung der Voraussetzungen gem. § 3 und der Vollständigkeit der Unterlagen legt der Dekan den Antrag unverzüglich dem Fachbereichsrat vor, der über die Zulassung zum Habilitationsverfahren beschließt sowie eine Habilitationskommission bildet und den Vorsitzenden der Kommission wählt. Die Zulassung zum Habilitationsverfahren wird dem Bewerber schriftlich mitgeteilt. (HO_21)

Ihre Reichweite betrifft andere Textteile (andere Paragraphen), sie zeigen die globale Kohäsion an, d.h. die Zusammenhänge zwischen makrostrukturellen Texteinheiten. In diesem Fall wird für sie die Bezeichnung makrostrukturelle Kohäsionsmittel / makrostrukturelle (= globale) Verweise vorgeschlagen. Sie ziehen sich durch den ganzen Text und viele seine Textsegmente hindurch. Exemplarisch soll das an einem Beispiel gezeigt werden (s. Abb. 1.).

Auch sie, wie unten an mehreren Belegen gezeigt wird, operieren in zwei Textrichtungen, sowohl anaphorisch als auch kataphorisch. Es lässt sich kein gravierender statistischer Unterschied in ihrer Verteilung feststellen. 


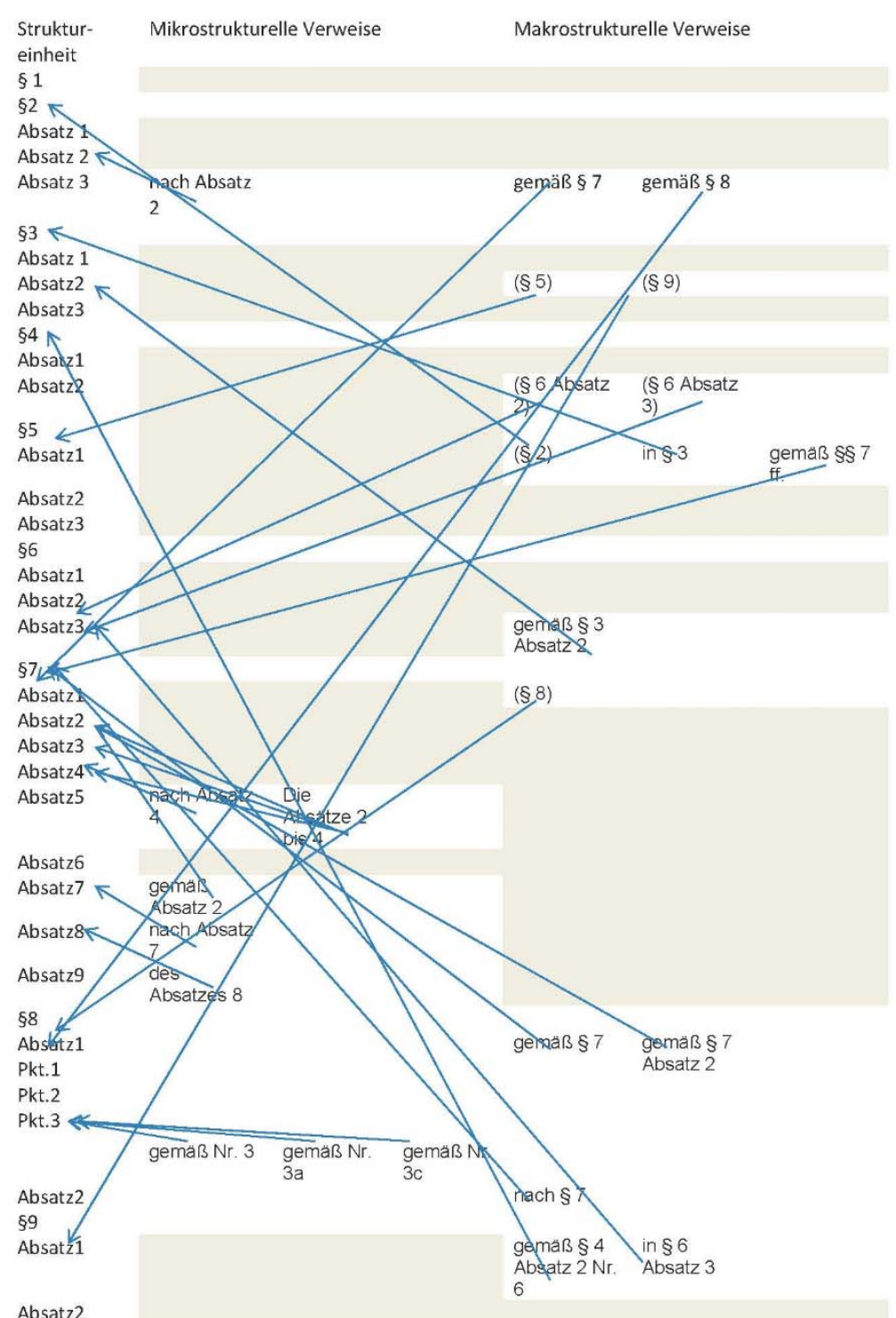

Abb. 1

Makrostrukturelle Verweise verbinden oft nicht nur einfach zwei Stellen miteinander, sondern sie erscheinen auch in Kaskadenform, d.h., es kommt eine Verweisung auf eine Textpassage zustande, die wiederum eine andere wieder aufgreift usw. So entsteht ein Netz von Verbindungen. Die Abbildung 2 illustriert schematisch die mögliche Verknüpfungsstruktur eines solchen Netzes. 


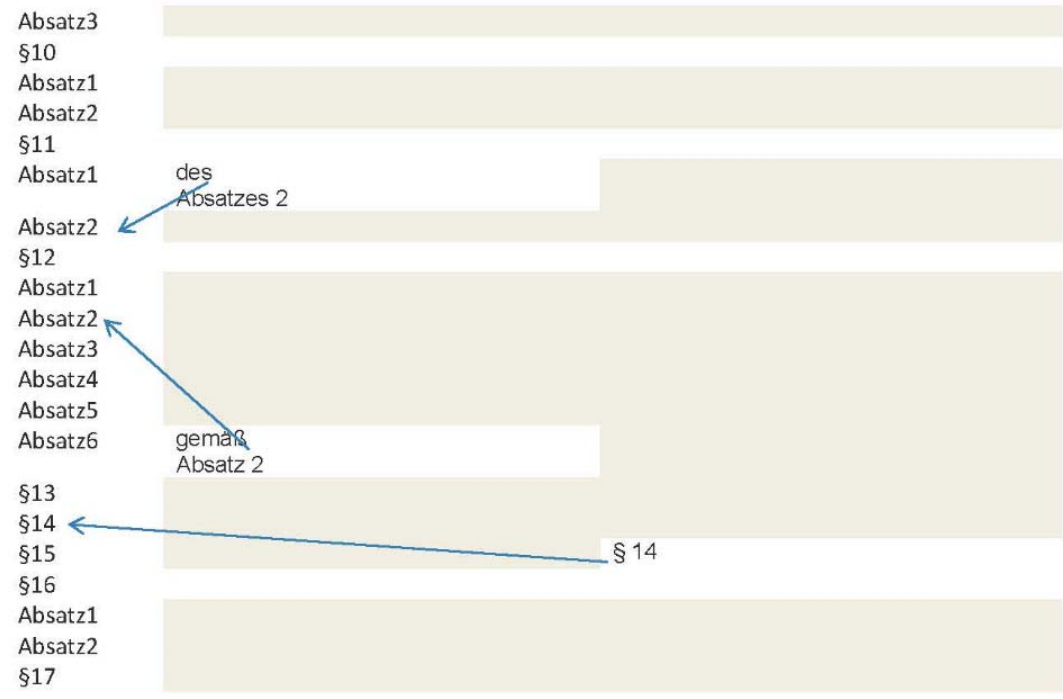

$A b b .2$

Eine solche Verfahrensweise führt vor Augen, was für ein kompliziertes und komplexes Gebilde ein Text trotz seiner materiellen Linearität darstellen kann. Solche Netze von Verknüpfungen dienen u.a. dazu, das jeweilige Textverknüpfungsmuster anzuzeigen. Die Habilitationsordnungen, deren globale Textkohärenz kaskadenartig erreicht wird, folgen dem argumentativ-explikativen Muster (vgl. Ch. Gansel/ F. Jürgens 2007: 137ff.). Die semantischen Relationen zwischen den verknüpften Stellen werden in einem anderen Abschnitt dieser Ausführungen kurz thematisiert.

\subsubsection{Zweistellige Relationen - mehrstellige Relationen}

Als ein weiteres Klassifikationskriterium kann die „Wertigkeit“ der Relationen herangezogen werden, die meisten Verweise (d.h. alle lokalen und die meisten globalen im untersuchten Material) stellen zweigliedrige (= einfache) Relationen her. Wie am Beispiel $28 \mathrm{zu}$ sehen ist, weist der Verweis auf eine bestimmte Textstelle /ein konkretes Bezugskonnekt, die / das mit der Ausgangsstelle / dem Trägerkonnekt zu verbinden sind.

(28) Im Falle einer Aufhebung erteilt der Dekan dem Habilitanden in angemessener Frist einen schriftlichen, mit Gründen und Rechtsbehelfsbelehrung gemäß Anlage 2 versehenen Bescheid. (HO_3)

Äußerst selten wird eine mehrstellige Relation angezeigt, es entsteht ein Verweisknoten. Dabei wird ein Bezug hergestellt zwischen dem Trägerkonnekt und mehreren Bezugsgrößen, die aneinandergereiht angegeben werden, die aber bei der Verarbeitung des Textes seitens des Rezipienten nicht linear wahrgenommen werden.

(29) $§ 3$ (1) Das Habilitationsgremium ist der Fachbereichsrat. Er führt das Habilitationsverfahren durch und entscheidet in allen Angelegenheiten, soweit nicht diese Ordnung etwas anderes vorsieht. Bei Entscheidungen des Fachbereichs nach $\S 6$ Abs. 1, 7 


\begin{abstract}
Abs. 6, $§ 9$ Abs. $1, \S 16$ und $\S 18$ sind nur Professoren und Habilitierte stimmberechtigt; mehr als die Hälfte dieser Mitglieder muß bei diesen Entscheidungen anwesend sein, die mit der Mehrheit der Anwesenden erfolgen; die übrigen Mitglieder des Fachbereichsrats wirken beratend mit. Bei Entscheidungen des Fachbereichsrats nach ist die Mehrheit der Professoren und Habilitierten des Fachbereichsrats berechtigt, wenn sie mit der Bestellung eines Gutachters durch die Mehrheit des Fachbereichsrats nicht einverstanden ist, zusätzlich einen weiteren Gutachter zu bestellen; auch bei dieser Abstimmung muß mindestens die Hälfte der stimmberechtigten Professoren und Habilitierten des Fachbereichsrates anwesend sein. (HO_15)
\end{abstract}

\title{
4.2.3. Semantische Relationen
}

Die durch Verweise verbundenen Stellen gehen unabhängig von ihrer "Wertigkeit" in eine Relation / Relationen ein, die sich auch semantisch charakterisieren lässt / lassen. Aus Platzgründen kann hier nicht auf alle im Untersuchungskorpus ermittelten Beziehungsarten eingegangen werden. Semantische Relationen können den Gegenstand eines separaten Beitrags bilden. Im Folgenden werden lediglich die unserer Ansicht nach für die Habilitationsordnung prototypischen Relationen untersucht, die in den analysierten Texten am häufigsten registriert werden konnten.

Eine Verweisung auf eine andere Stelle im Text mit ihrem semantischen Gehalt bedeutet eine semantische Ergänzung der Ausgangsstelle. Das Trägerkonnekt ist demnach für die Rezeption des Textes semantisch nicht vollständig und daher ergänzungsbedürftig. Dabei muss hervorgehoben werden, dass solche Texte wie Ordnungen in der Regel selektiv rezipiert werden. Der Leser sucht sich nach Interesse und Ziel entsprechende Textabschnitte heraus. Der Verweis, den eine Stelle enthält, sorgt für die Vervollständigung der Informationen.

In unserem Untersuchungskorpus lassen sich folgende semantische Relationen ermitteln:

\section{- additive Relation}

(30a) Werden an Stelle der Habilitationsschrift gemäß § 4 Absatz 1 Nr. 1 eine oder mehrere wissenschaftliche Veröffentlichungen gemäß $§ 4$ Absatz 2 vorgelegt, müssen sie einzeln oder in ihrer Gesamtheit den Anforderungen nach Absatz 1 genügen. (HO_29)

(30b) Die Habilitationsschrift muss dem Wissenschaftsgebiet entstammen, für welches der Bewerber die Anerkennung der Lehrbefähigung anstrebt. Sie muss einen wesentlichen Beitrag zum wissenschaftlichen Fortschritt darstellen, selbständig erarbeitet sein und eine besondere Befähigung zu selbständiger Forschungstätigkeit erkennen lassen. (HO_29)

Die Additivität kann z.B. an Belegen (30a) und (30b) beobachtet werden. In (30a) werden Anforderungen an die Habilitationsschrift erwähnt, die in (30b) der Reihe nach (additiv) aufgezählt werden. Durch eine solche Verfahrensweise erreicht man eine kompakte und leicht zu überblickende Ausformulierung, die an der mit dem Verweis angezeigten Stelle spezifiziert wird. 
(31a) (1) Die Dekanin oder der Dekan prüft die gemäß § 4 von der Bewerberin oder dem Bewerber vorgelegten Unterlagen auf Vollständigkeit und beruft den Fakultätsrat zur Bestellung der Mitglieder einer Habilitationskommission ein.

(31b) § 4 Habilitationsantrag

(1) Der Antrag auf Zulassung zur Habilitation und damit der Eröffnung des Habilitationsverfahrens ist schriftlich

an die Dekanin oder den Dekan der Philosophischen Fakultät zu richten. In dem Antrag hat die Bewerberin oder der Bewerber anzugeben, für welches Fachgebiet oder Fach sie oder er die Lehrbefugnis erwerben will.

(2) Dem Antrag auf Zulassung zur Habilitation müssen beigefügt werden:

1. ein Lebenslauf mit Darstellung des persönlichen und beruflichen Werdegangs,

2. eine beglaubigte Kopie der Promotionsurkunde,

3. sonstige akademische Urkunden und Zeugnisse in beglaubigter Form,

4. ein vollständiges Verzeichnis der wissenschaftlichen Veröffentlichungen,

5. eine Übersicht über die bisherige Lehrtätigkeit,

6. die Habilitationsschrift oder andere wissenschaftliche Veröffentlichungen gemäß

3, Absatz 3 in vier Exemplaren,

7. je eine Zusammenfassung in deutscher und englischer Sprache,

8. eine Erklärung über bisher unternommene Habilitationsversuche,

9. ein polizeiliches Führungszeugnis, das nicht älter als ein Jahr ist und eine Mitteilung über anhängige

Strafverfahren,

10. eine Erklärung der Bewerberin oder des Bewerbers, dass bei der Anfertigung der schriftlichen Habilitationsleistung

kein wissenschaftliches Fehlverhalten im Sinne der Richtlinie der Gottfried Wilhelm Leibniz Universität

Hannover zur Sicherung guter wissenschaftlicher Praxis in der jeweils gültigen Fassung vorliegt,

11. drei Themenvorschläge für den wissenschaftlichen Vortrag mit Kolloquium,

12. ein Selbstbericht zur Lehre, der folgende Unterlagen enthält:

a) Verzeichnis der durchgeführten Lehrveranstaltungen

b) Evaluationsberichte über die evaluierten Lehrveranstaltungen

c) Dokumentation einer selbstverantwortlich durchgeführten Lehrveranstaltung.

(3) Der Antrag und die Anlagen verbleiben nach Abschluss des Verfahrens bei der Fakultät.

(4) Über den Antrag auf Zulassung zur Habilitation sind die habilitierten oder gleichwertig qualifizierten Mitglieder der Fakultät zu informieren. (HO_17)

Mit einem ähnlichen Fall haben wir es bei Belegen (31a) und (32b) zu tun: eine eingangs in (31b) genannte Information dient der Ergänzung und Präzisierung einer weiteren, die durch den Verweis in (2a) sehr prägnant und ohne Wiederholungen formuliert werden konnte.

- konditionale Relation

(32a) Voraussetzung ist, dass die für das Habilitationsverfahren eines der Autorinnen oder Autoren zu berücksichtigenden Beiträge zweifelsfrei dieser Bewerberin oder diesem Bewerber zugerechnet werden können und den Anforderungen nach $\S 1$ Abs. 1 sowie Abs. 1 genügen. 
(32b) (1) 1 Die Bewerberin oder der Bewerber muss den Doktorgrad an einer deutschen wissenschaftlichen Hochschule oder einer gleichwertigen ausländischen Hochschule erworben haben. 2 Ihre oder seine Befähigung zu selbständiger wissenschaftlicher Forschung wird durch eine Habilitationsschrift nachgewiesen. 3 Anstelle einer Habilitationsschrift können bisherige Veröffentlichungen und zur Veröffentlichung angenommene Arbeiten anerkannt werden, wenn sie einen gleichwertigen Nachweis der Befähigung zur selbständigen wissenschaftlichen Forschung darstellen und noch nicht in einem Habilitationsverfahren verwendet wurden. (HO_18)

In (32a) kommen Anforderungen und Voraussetzungen zur Sprache, denen der Bewerber / die Bewerberin entsprechen muss, um zu weiteren Etappen des Habilitationsverfahrens zugelassen zu werden. Zwischen dem Verweis in (32a) und dem Beleg (32b) besteht u.E. eine konditionale Relation, die sprachlich u.a. durch das Modalverb müssen ausgedrückt wird. Nicht ohne Bedeutung ist in dem Zusammenhang der spezifische Gebrauch vom Indikativ (wird... nachgewiesen), der dem sprachlichen Vollzug einer Verpflichtung dient. Eine weitere Kennzeichnung der Konditionalität erfolgt durch das Nomen Voraussetzung.

(33b) (1) Die Lehrbefähigung erlischt, wenn der/die Habilitierte den Doktorgrad nicht mehr führen darf. Die Feststellung des Erlöschens trifft der Präsident/die Präsidentin der Humboldt-Universität zu Berlin auf Antrag der Theologischen Fakultät (§ 36, Absatz 7 BerlHG).

(2) Die Lehrbefähigung wird durch Beschluss des erweiterten Fakultätsrates zurückgenommen, wenn die Habilitation erschlichen oder sonst mit unlauteren Mitteln erlangt worden ist.

(33a) (3) In Fällen von Absatz 1 und 2 ist die Habilitationsurkunde einzuziehen. (HO_9)

Eine eindeutige und nur durch das verweisende Element erzielte Konditionalität liegt im Beleg 33 vor. Hier werden Bedingungen (33b) angeführt, unter denen ein Prozess (33a) vollzogen werden kann.

\section{- definitorische Relation}

(34a) (1)Nach der Zulassung zum Habilitationsverfahren bestimmt die dem Habilitationsausschuss vorsitzende Person im Benehmen mit der Bewerberin bzw. dem Bewerber die studiengangbezogene Lehrveranstaltung, die dem Nachweis der pädagogisch-didaktischen Eignung dienen soll. Als studiengangbezogene Lehrveranstaltung gilt jede mindestens zwei Semesterwochenstunden umfassende Veranstaltung im Sinne eines Studienplans des Fachs oder Fachgebiets, in dem die Habilitation angestrebt wird.

(34b) Sobald eine Veranstaltung im Sinne von Absatz 1 bestimmt ist, zeigt die dem Habilitationsausschuss vorsitzende Person dies den Mitgliedern des Habilitationsausschusses schriftlich an. Die Frist zwischen dieser Mitteilung und dem Beginn der Veranstaltung soll mindestens zwei Wochen betragen. (HO_8)

(35a) Solange der Dekanin oder dem Dekan noch kein Gutachten im Sinne des $§ 7$ vorliegt, kann die Bewerberin oder der Bewerber ohne Angaben von Gründen vom Verfahren zurücktreten. Als Zeitpunkt des Rücktritts gilt der Eingang des Rücktrittsgesuchs bei der Dekanin oder dem Dekan der Fakultät. (HO_17) 
(35b) § 7 Begutachtung der schriftlichen Habilitationsleistung

(1) Wird das Habilitationsverfahren eröffnet, bestellt die Habilitationskommission mindestens zwei habilitierte oder gleichwertig qualifizierte Gutachterinnen oder Gutachter zur Beurteilung der schriftlichen Habilitationsleistung. Im Laufe des Verfahrens können weitere Gutachterinnen oder Gutachter bestellt werden. Zur Gutachterin oder zum Gutachter kann nur bestellt werden, wer die durch die Habilitationsschrift angestrebte Lehrbefugnis, die Lehrbefugnis eines fachnahen Gebiets oder Spezialkenntnisse zur Beurteilung besonderer Aspekte der Habilitationsschrift besitzt. Die Frist für die Erstellung der Gutachten beträgt in der Regel drei Monate. Bei Fristüberschreitung kann eine neue Gutachterin oder ein neuer Gutachter bestellt werden.

(2) Die Gutachten müssen auf Grundlage der eigenen, unmittelbaren und vollständigen Kenntnisnahme der Habilitationsschrift eine fachwissenschaftliche Beurteilung enthalten sowie zu einer schlüssigen Bewertungsentscheidung über die Annahme oder Ablehnung der Arbeit führen. Die Gutachten sind eingehend zu begründen.

(3) Die Gutachten sowie die schriftliche Habilitationsleistung werden den Mitgliedern der Habilitationskommission und den habilitierten oder gleichwertig qualifizierten Mitgliedern der Fakultät durch Auslage im Dekanat oder anderweitig bekannt gegeben. Eine Sitzung der Habilitationskommission zur Entscheidung über die schriftliche Habilitationsleistung darf erst nach Ablauf einer vierwöchigen Einsichtnahmefrist erfolgen.

In den obigen Belegen wird jeweils eine für das Habilitationsverfahren zentrale Entität angeführt, die in der Regel unterschiedliche Interpretationen zulässt, so dass ihre Bedeutung in dem Kontext als polysem empfunden werden kann. Um dem vorzubeugen und eine Eindeutigkeit zu erzielen, bedienen sich die Autoren der HOs einer Verweisprozedur, die die jeweilige vollständige Bedeutung bestimmt. Dies sehen wir als einen Versuch an, die Entität zu definieren. In (34b) ist von einer Veranstaltung die Rede, die in (34a) ausführlich und präzise mit vielen Merkmalen bestimmt wird. Ähnliches lässt sich im Falle des Gutachtens in (35a) beobachten. Das Gutachten kann hier als ein Prozess aufgefasst werden, dessen Modalitäten (Beteiligte, Fristen etc.) in (35b) detailliert festgelegt werden.

Nach unserem Dafürhalten bestehen Unterschiede zwischen solchen definitorischen und den oben dargestellten additiven Beziehungen: im ersteren Falle wird ein Begriff definiert und abgegrenzt, das macht ihn erst einmal für den Leser nachvollziehbar, im letzteren Fall werden die Komponenten der Bedeutung im gewissen Sinne "aufgerollt", nacheinander aufgezählt, erweitert, präzisiert, spezifiziert etc.

Die in juristischen Texten, auch in Habilitationsordnungen geschilderten Sachverhalte sind durch eine große Komplexität gekennzeichnet, eine Habilitationsordnung beschreibt und legt einen komplexen Vorgang fest, der sich aus mehreren Etappen zusammensetzt. Auch die einzelnen Phasen charakterisieren sich durch ihre Eigendynamik, die in aller Detailliertheit in der Ordnung geregelt werden soll. Die Etappen sind auch aufs engste miteinander verbunden. Dieser Tatsache muss auch die Textgestaltung Rechnung tragen und sie manifestiert sich im Textaufbau, seiner Struktur und seiner formalen Ausgestaltung. Diese Aufgabe übernimmt auch die sog. globale Textkohäsion. Als ihre Ausdrucksmittel fungieren beispielsweise Überschriften. Wir vertreten die These, dass auch juristische intratextuelle Verweise als Elemente der globa- 
len Textkohäsion aufgefasst und dementsprechend linguistisch analysiert und beschrieben werden sollen. Sie stellen Verknüpfungen zwischen einzelnen Textabschnitten und innerhalb eines Textsegmentes her. Sie operieren anaphorisch und (seltener) kataphorisch und verbinden die genannten Textstellen, indem sie diverse Relationen zwischen ihnen einerseits herstellen und andererseits anzeigen. Ihre Leistungen können unserer Meinung nach ähnlich wie die der syntaktischen Konnektoren geschildert werden. Den Konnektoren im Deutschen wurden zahlreiche sprachwissenschaftliche Arbeiten (R. Pasch/ U. Brauße/ E. Breindl/ U.H. Waßner (2003) ${ }^{11}$ gewidmet. Einen neuen Ansatz präsentiert in einer Reihe von Aufsätzen H. Blühdorn (2008, 2010 und andere), dessen Auffassung der semantischen Verknüpfungsrelationen den Ausgangspunkt für unsere Analyse darstellt. Da graphische Verweise, wie gesagt, ähnlich wie Konnektoren zwei Stellen im Text in eine Relation bringen, wollen wir überprüfen, ob sich die Art und Weise dieser Relationen mit dem bei Blühdorn in Bezug auf syntaktische konnektive Mittel zusammengestellten Kategorien erschöpfend beschreiben lässt.

H. Blühdorn (2008: 33ff.) spricht von vier Kategorien der semantischen Relationen von Konnektoren: Ähnlichkeits-, Situierungs-, Konditional- und Kausalrelationen. Im Falle von Ähnlichkeitsrelationen besteht eine Symmetrie zwischen den in Relation eingehenden Elementen, sie weisen gleiches semantisches Gewicht auf, sie sind sozusagen zueinander alternativ. Die übrigen drei Relationsklassen sind asymmetrisch. Bei den Situierungsrelationen ,wird eines der Relata in der relevanten Domäne relativ zum anderen verortet" (H. Blühdorn 2010: 208). Bei der Kohäsionsrelation vom Typ Situierung wird der zu situierende Relat durch das andere Glied in einer Domäne unterschiedlicher Art platziert. Konditionalrelationen lassen sich ganz allgemein als Bedingung-Folge-Relationen und Kausalrelationen, bekanntlich als Ursache-Wirkung-Folgen charakterisieren. Im Rahmen der Ähnlichkeitsrelation werden komparative, disjunktive auch additive Relationen genannt. Die Situierungsrelationen umfassen (modal) adversative, temporale und lokale und die Konditionalrelationen finale, instrumentale, konzessive und konditionale Relationen. $\mathrm{Zu}$ der Menge der Kausalrelationen gehören konklusive, evidentielle, instrumentale, konzessive, konsekutive und kausale Relationen.

Klassifikation nach der Reichweite innerhalb des Textes

1. Verweise innerhalb eines Abschnitts /Paragraphen

Als Ausdruck der lokalen bzw. mikrostrukturellen Kohäsion fungieren Bezüge innerhalb eines Textsegments. Sie kommen in Abschnitten vor, die in Absätze gegliedert sind und ausschließlich anaphorisch operieren. Auf den im eingangs eingeführten Ansatz dargestellten Sachverhalt wird im zweiten zurückgegriffen. Die Gestaltung eines solchen Verweises nimmt die Form einer einfachen Präpositionalphrase an, entweder mit der lokalen Präposition in [(2) Wird eine der in Absatz 1 bezeichneten Leistungen als ,nicht ausreichend" beurteilt, so ist die gesamte Habilitation nicht bestanden. HO_19)] oder der modalen Präposition nach [(6) Wird die mündliche Habilitationsleistung nach Abs. 3 als nicht den Anforderungen entsprechend bewertet, so ist das Habilitationsverfahren beendet. (HO_27)].

\footnotetext{
${ }^{11} \mathrm{http}: /$ hypermedia.ids-mannheim.de/call/public/sysgram.ansicht?v_typ=d\&v_id=366, letzter Zugriff am 16.02.2017.
} 
2. Verweise außerhalb eines Textsegments

In einem solchen Fall liegt die globale (makrostrukturelle) Textkohärenz vor, es werden Bezüge über den Text hindurch gezogen. Dank ihnen kommen Bezüge in zwei Richtungen zustande, sie verweisen sowohl anaphorisch als auch kataphorisch, wobei in den analysierten Texten kataphorische Verweise häufiger auftreten, z.B.

\section{§ 2 Habilitationsleistungen}

Die Habilitation umfaßt Leistungen in Forschung und Lehre. Diese Leistungen werden durch die in $\S \mathbf{4}$ genannten Vorleistungen, eine öffentliche Lehrprobe, die Habilitationsschrift und einen Vortrag mit wissenschaftlicher Aussprache (Disputation) erbracht.

\section{$\S 3$ Habilitationsgremium}

(1) Das Habilitationsgremium ist der Fachbereichsrat. Er führt das Habilitationsverfahren durch und entscheidet in allen Angelegenheiten, soweit nicht diese Ordnung etwas anderes vorsieht. Bei Entscheidungen des Fachbereichs nach § 6 Abs. 1, § 7 Abs. 6, § 9 Abs. 1, § 16 und § 18 sind nur Professoren und Habilitierte stimmberechtigt; mehr als die Hälfte dieser Mitglieder muß bei diesen Entscheidungen anwesend sein, die mit der Mehrheit der Anwesenden erfolgen; die übrigen Mitglieder des Fachbereichsrats wirken beratend mit. Bei Entscheidungen des Fachbereichsrats nach $\$ 7$ Abs. 1 und 4 ist die Mehrheit der Professoren und Habilitierten des Fachbereichsrats berechtigt, wenn sie mit der Bestellung eines Gutachters durch die Mehrheit des Fachbereichsrats nicht einverstanden ist, zusätzlich einen weiteren Gutachter zu bestellen; auch bei dieser Abstimmung muß mindestens die Hälfte der stimmberechtigten Professoren und Habilitierten des Fachbereichsrates anwesend sein. (HO_15)

Anaphorische Verweise:

\section{§ 9 Mündliche Prüfung}

(2) Nach Annahme der Habilitationsschrift wird vom Habilitationsausschuß aus drei Vorschlägen des Bewerbers das Thema für den öffentlichen Vortrag ausgewählt. Der wissenschaftliche Vortrag soll ein Thema des Faches oder Fachgebiets behandeln, für das die Lehrbefähigung angestrebt wird. Die Themenvorschläge müssen sich von den im Verzeichnis der wissenschaftlichen Arbeiten gemäß $\$ 4$ Abs. 1, Ziffer 4 angeführten Themenkreise unterscheiden. Der Habilitationsausschuß kann weitere Themenvorschläge vom Bewerber verlangen. Das ausgewählte Thema und der Termin sind dem Bewerber mindestens zwei Wochen vor dem Vortrag mitzuteilen. (HO_27)

Abgesehen von der Richtung des Operierens unterscheiden sich makrostrukturelle anaphorische und kataphorische Verweise weder in der Form noch in der Art der Relation, in die die zwei verbundenen Textpassagen eingehen.

Das Verhältnis zwischen den beiden verknüpften Stellen lässt sich folgendermaßen graphisch wiedergegeben:

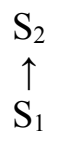

Die erste Konstituente (der 1. Absatz) ist der zweiten untergeordnet und ihre Leistung besteht darin, die Informationen der zweiten zu ergänzen, indem eine Definition eines im zweiten Abschnitt genannten Phänomens geliefert wird. Diese Relation wird hier als DEFINIEREN bezeichnet. 


\section{Zusammenfassung und Fazit}

Die analysierten Textbezüge halten wir für ein Spezifikum der Rechtstexte, die zweifelsohne zu wichtigen kohärenzstiftenden Mitteln gehören, die außer in Rechtstexten und wissenschaftlichen Arbeiten kaum anzutreffen sind. Das Textverstehen ist ein Interpretationsvorgang, der schrittweise erfolgt. Die gesamte Bedeutung des Textes wird im Spannungsfeld von Wissen, Erwartungen und Überzeugungen (vgl. Klitsch_1988) erschlossen. Den Prozess erleichtern und begünstigen textoberflächliche Mittel, denen formale Verweise angehören.

Bei Habilitationsordnungen muss zusätzlich beachtet werden, dass Leser in der Regel bestimmte Textpassagen (Textinhalte) entsprechend ihren Interessen / Bedürfnissen auswählen. Die Verweise tragen in einem solchen Fall dazu bei, dass das nötige Wissen durch Bezüge auf andere Textstellen relativ vollständig vermittelt wird und dadurch das individuelle Leseziel des Rezipienten erreicht wird.

Formal gesehen dominieren PPs als Verweisexponenten, gefolgt von Quasi PPs wie im Sinne. Von den syntaktischen Funktionen solcher Phrasen wurde im untersuchten Material fast ausschließlich das Attribut verschiedenen Grades festgestellt, viel seltener wird die adverbiale Funktion realisiert, mitunter ließ sich nur unter Heranziehung des Kontextes entscheiden, ob im gegebenen Fall ein Attribut oder Adverbial vorliegt.

Die meisten Verweise sind kataphorisch, anaphorische treten viel seltener auf. Beide wurden in mikro- und makrostrukturelle Verweise eingeteilt: Die ersteren operieren innerhalb einer Texteinheit, z.B. eines Paragraphen, und sind als Ausdruck der lokalen Kohäsion aufzufassen, während die letzteren über die Paragraphengrenzen hinausgehen und die globale Kohäsion anzeigen.

Je nachdem, ob sich das Trägerkonnekt auf eine oder auf mehrere Stellen im Text bezieht, haben wir einstellige und mehrstellige Relationen unterschieden. Die letzteren unterscheiden sich von den ersteren, dass das Trägerkonnekt gleichzeitig auf mehrere Bezugsgrößen verweist, infolge dessen nestförmige Strukturen mit dem Trägerkonnekt im Zentrum entstehen. Das Träger- und Bezugskonnekt gehen auch spezifische semantische Relationen ein, und zwar die (i) additive, (ii) konditionale, (iii) definitorische Relation. So gestalten sich im Großen und Ganzen die intratextuellen Verweise in der Textsorte Habilitationsordnung. Interessante Ergebnisse könnte auch die Untersuchung der intertextuellen Verweise liefern, was ein Textsortennetz voraussetzen müsste. Dies möchten wir jedoch künftiger Forschung überlassen.

\section{Literaturverzeichnis}

Adamzik, K. (1995), Textsorten - Texttypologie. Eine kommentierte Bibliographie. Münster.

de Beaugrande, R./ W.U. Dressler (1981), Einführung in die Textlinguistik (Konzepte der Sprach- und Literaturwissenschaft 28). Tübingen.

Blühdorn, H. (2008), Syntax und Semantik der Konnektoren. Ein Überblick. Mannheim. Manuskript. (URL http://www.ids-mannheim.de/gra/texte/blu_ ueberblick.pdf). 
Blühdorn, H. (2010), Semantische Unbestimmtheit bei Konnektoren. In: I. Pohl (Hrsg.), Semantische Unbestimmtheit im Lexikon. Frankfurt a. M., 205-221.

Brinker, K. (2001), Linguistische Textanalyse. Eine Einführung in Grundbegriffe und Methoden. 5. Auflage, (Grundlagen der Germanistik 29). Berlin.

Busse, D. (2000), Textsorten des Bereichs Rechtswesen und Justiz. In: G. Antos/ K. Brinker/ W. Heinemann/ S.F. Sager (Hrsg.), Text- und Gesprächslinguistik. Ein internationales Handbuch zeitgenössischer Forschung (Handbücher zur Sprachund Kommunikationswissenschaft). Berlin/ New York, 658-675.

Di Meola, C. (1999), Entgegen, nahe, entsprechend und gemäß. Dativpräpositionen mit Genitivrektion. In: „Zeitschrift für Germanistische Linguistik“ 27, 344-351.

Di Meola, C. (2004), The rise of the prepositional genitive in German - a grammaticalization phenomenon. In: „Lingua“ 114, 165-182.

Duden (2006), Duden. Die Grammatik. 7. Auflage. Mannheim.

Duden (2009), Duden. Die Grammatik. 8. Auflage. Mannheim.

Duden (2001), Deutsches Universalwörterbuch. 4. Auflage. Mannheim.

Duden (2001), Richtiges und gutes Deutsch. Wörterbuch der sprachlichen Zweifelsfälle. 5. Auflage. Mannheim.

Engberg, J. (1993), Prinzipien einer Typologisierung juristischer Texte. In: „Fachspra-

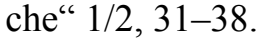

Engberg, J. (1997), Konventionen von Fachtextsorten - Kontrastive Analysen zu deutschen und dänischen Gerichtsurteilen. Tübingen.

Engel, U. (1988), Deutsche Grammatik. Heidelberg.

Fandrych, Ch./ M. Thurmair (2011), Textsorten im Deutschen. Linguistische Analysen aus didaktischer Sicht. Tübingen.

Fritz, T.A. (2005), Der Text. In: M. Wermke/ K. Kunkel-Razum/ W. Scholze-Stubenrecht (Hrsg.), DUDEN. Die Grammatik. Unentbehrlich für richtiges Deutsch. (Bd. 4, 7. Aufl., 1067-1174). Mannheim.

Gansel, Ch./ F. Jürgens (2007), Textlinguistik und Textgrammatik. Eine Einführung. Göttingen.

Halliday, M.A.K./ H. Ruqaiya (1976), Cohesion in English. London.

Handbuch der Rechtsförmlichkeit (2008), (URL.http://hdr.bmj.de/vorwort.html).

Helbig, G./ J. Buscha (1994), Deutsche Grammatik. Ein Handbuch für den Ausländerunterricht. Leipzig etc.

Hertel, L./ B. Oberbichler/ Th. Wilrich (2015), Technisches Recht. Grundlagen - Systematik-Recherche. Berlin etc.

Klitsch, W. (1988), The role of knowledge in discourse comprehension: a constructionintegration model. In: „Psychological review” 95, 163-182.

Koch, P./ W. Oesterreicher (1994), Schriftlichkeit und Sprache. In: H. Günther/ O. Ludwig (Hrsg.), Schrift und Schriftlichkeit. Writing and Its Use. Ein interdisziplinäres Handbuch internationaler Forschung. An Interdisciplinary Handbook of International Research. Berlin/ New York, 587-604.

Linke, A./ M. Nussbaumer/ P.R. Portmann (2004), Studienbuch Linguistik. 5. erw. Auflage, Tübingen.

Mikołajczyk, B./ J. Aptacy (2015a), „Das Gesuch um Zulassung zur Habilitation ist an den Dekan zu richten ". Sprachliche Realisierung des VERPFLICHTENs in Habilitationsordnungen. Dargestellt an den Habilitationsordnungen der Christian- 
Albrechts-Universität zu Kiel. In: M. Wierzbicka/ B. Rolek (Hrsg.), Grammatische Strukturen im Text und im Diskurs, Bd.4, Rzeszów, 53-76.

Mikołajczyk, B./ J. Aptacy (2015b), Fachtermini in der Rechtssprache - Lehrbefugnis und Lehrbefähigung im Hochschulrecht. Zur Bedeutung und Verwendung der Fachwörter in der Textsorte Habilitationsordnung. In: „Studia Linguistica“ 34, 91-115.

Mikołajczyk, B./ J. Aptacy (2017, im Druck), Terminologia specjalistyczna w zakresie niemieckiego prawa o szkolnictwie wyższym (na przykładzie niemieckich Lehrbefähigung/ Lehrbefugnis) jako wyzwanie dla tłumacza na język polski. Rozważania lingwistyczne. In: A. Fimiak-Chwiłkowska/ B. Walkiewicz/ J. Woroch (Hrsg.), Norma a uzus I. Z zagadnień przekładu specjalistycznego.

Möhn, D. (2001), 'weil es notwendig ist daß jeder eines anderen Pflichten und Befugnisse kennt. 'Johann Wolfgang von Goethe als Ordnungstextautor. In: C. Mauelshagen/ J. Seifert (Hrsg.), Sprache und Text in Theorie und Empirie. Beiträge zur germanistischen Sprachwissenschaft. Festschrift für Wolfgang Brandt. (,Zeitschrift für Dialektologie und Linguistik, Beihefte", Bd.114), 55-70.

Morlok, M. (2015), Intertextualität und Hypertextualität im Recht. In: F. Vogel (Hrsg.), Zugänge zur Rechtssemantik. Interdisziplinäre Ansätze im Zeitalter der Mediatisierung, 69-90.

Mushchinina, M. (2017), Sprachverwendung und Normvorstellung in der Fachkommunikation (Forum für Fachsprachen-Forschung 132). Berlin.

Niedobitek, M. (2001), Das Recht der grenzüberschreitenden Verträge. Tübingen.

Pasch, R./ U. Brauße/ E. Breindl/ U.H. Waßner (2003), Handbuch der deutschen Konnektoren. Linguistische Grundlagen der Beschreibung und syntaktische Merkmale der deutschen Satzverknüpfer (Konjunktionen, Satzadverbien und Partikeln). Berlin/ New York.

Paulfranz, A. (2013), Kasusmarkierungen der Gegenwartssprache in deutschen Lokalund Regionaltageszeitungen. Bamberg.

Rickheit, G./ U. Schade (2000), Kohärenz und Kohäsion. In: K. Brinker et al. (Hrsg.), Text- und Gesprächslinguistik. Ein internationales Handbuch zeitgenössischer Forschung. Volume 1. (Handbücher zur Sprach- und Kommunikationswissenschaft 16.1). Berlin/ New York, 275-283.

Schmitz, A. (2016), Verständlichkeit von Sachtexten: Wirkung der globalen Textkohäsion auf das Verständnis von Schülern. Wiesbaden.

Vater, H. (2007), Kasusgebrauch im gegenwärtigen Deutschen. In: „Estudios Filológicos Alemanes" 14, 9-46.

Weinrich, H. (1993), Textgrammatik der deutschen Sprache. Mannheim.

\section{Internetquellen}

(URL http://www.deutschegrammatik20.de/praepositionen/die-bedeutung-der-prapositionen-ubersicht/die-bedeutung-der-prapositionen-gemas/). [letzter Zugriff am 13.11.2016].

(URL .http://hypermedia.ids-mannheim.de/call/public/sysgram.ansicht? v_typ $=d \& v_{-}$id $\left.=366\right)$. [letzter Zugriff am 13.11.2016]. 